\title{
Kinetics of Proton Transport in Water
}

\author{
A. A. Kornyshev, ${ }^{*, \dagger, \$}$ A. M. Kuznetsov ${ }^{\dagger, \S, \|}$ E. Spohr, ${ }^{\dagger}$ and J. Ulstrup ${ }^{\perp}$ \\ Institute for Materials and Processes in Energy Systems (IWV3), Research Center Jülich, \\ D-52425 Jülich, Germany; Department of Chemistry, Faculty of Physical Sciences, Imperial \\ College London, London SW7 2AZ, U.K.; The A. N. Frumkin Institute of Electrochemistry, \\ Russian Academy of Sciences, 117071 Moscow, Russia; Department of Chemistry, \\ The Technical University of Denmark, DK-2800 Lyngby, Denmark; and \\ Department of Electrochemistry, University of Ulm, D-89069 Ulm, Germany
}

Received: April 1, 2002; In Final Form: August 22, 2002

\begin{abstract}
The excess proton mobility in water has attracted scientific attention for more than a century. Detailed theoretical concepts and models are also presently in strong focus in efforts toward understanding this ubiquitous phenomenon. In the present report, we discuss a theoretical framework for rationalizing the excess proton mobility, based on computer simulations, theory of proton transfer (PT) in condensed media, and analysis of classical proton conductivity experiments over broad temperature ranges. The mechanistic options involved are (i) classical hydrodynamic motion of the hydronium ion $\left(\mathrm{H}_{3} \mathrm{O}^{+}\right)$, (ii) proton transfer from hydronium to a neighboring water molecule, and (iii) structural diffusion of the Zundel complex $\left(\mathrm{H}_{5} \mathrm{O}_{2}{ }^{+}\right)$, the processes all controlled by orientational fluctuations or hydrogen bond breaking in neighboring hydration shells. Spontaneous conversion of excess proton states between Zundel and hydrated hydronium states and between hydrated and bare hydronium states are the crucial parts of the scheme. A comparison between experimental data and molecular dynamics (MD) simulations shows that prototropic structural diffusion is determined by comparable contributions of the Zundel and hydrated hydronium states. The temperature dependent mobility is, moreover, determined not only by activation free energies of the three different acts of charge transfer, but also by labile equilibria between the different PT clusters. The proton conduction mechanisms of the three clusters are brought into the framework of quantum mechanical PT theory in condensed media. Both the nature of the elementary act and the reaction coordinates are, however, different for the two types of PT clusters. The corresponding rate constants are calculated and compared with MD simulations. Within the framework of PT theory we can also identify the nature of the kinetic deuterium isotope effect in the strongly interacting proton donor and acceptor groups in the clusters. The views and models introduced may carry over to PT in more composite, heterogeneous, and confined environments such as in polymer electrolyte membrane systems.
\end{abstract}

\section{Introduction}

Water is an excellent conductor for excess protons. The conductance of distilled water was never an issue, per se, because the number of free protons is very small $\left(10^{-7} \mathrm{~mol} / \mathrm{L}\right)$. However, the proton mobility in water is approximately five times higher than the mobility of an alkali cation (e.g., $\mathrm{Na}^{+}$) of similar size as the hydronium $\left(\mathrm{H}_{3} \mathrm{O}^{+}\right)$ion. ${ }^{1}$ Excess protons in aqueous phases are thus highly mobile. This happens, e.g., in acid solutions or in hydrated polymer-electrolyte membranes or proteins. In solutions of strong acids, both the protons and counteranions are mobile. The counteranions in membranes and proteins are mostly part of an immobile skeleton, with protons moving through water-filled channels in the skeleton.

* To whom correspondence should be addressed. E-mail: a.kornyshev@ ic.ac.uk.

Institute for Materials and Processes in Energy Systems.

$\doteqdot$ Imperial College London.

$\S$ Russian Academy of Sciences.

" University of Ulm.

${ }^{\perp}$ The Technical University of Denmark.
Experimental studies of temperature-dependent proton mobility have a long history. In a modern sense, they date back to work by Johnston ${ }^{2}$ and Noyes. ${ }^{3}$ This work was extended, including studies of the pressure dependence and kinetic deuterium isotope effects by Eucken, ${ }^{4}$ Gierer and Wirtz, ${ }^{5}$ Gierer, ${ }^{6}$ and Franck, Hartmann, and Hensel. ${ }^{7}$ Reference 8 gives a comprehensive overview of aqueous proton conductivity and the early experimental data. The discussion in these works is based on the so-called excess mobility (see below). It is of importance for the following discusion that the excess mobility vs temperature curve was found to exhibit a maximum at temperatures near $150^{\circ} \mathrm{C}$, at elevated pressure. The net value of the proton mobility in pure water was not addressed in those studies, although attempts to determine it were made by Kohlrausch at the end of the 19th century. ${ }^{9}$ Focus was instead on the conductance of strong acids such as $\mathrm{HCl}$ in the limit of infinite dilution. The difference of the measured conductance and the limiting conductance of a salt of a cation with similar size as $\mathrm{H}_{3} \mathrm{O}^{+}$was attributed to excess proton mobility, based 
on the assumption that the hydrodynamic radius of both ions would be similar. The excess mobility was taken to represent nonclassical proton hops in addition to classical hydrodynamic motion of the $\mathrm{H}_{3} \mathrm{O}^{+}$ion.

Proton conductivity in bulk aqueous solution can be contrasted with proton conductance in water-saturated polymer electrolyte membranes, such as perfluorinated sulfonic acids. ${ }^{10,11,12}$ These materials are important from a fundamental point of view because they have only single charge carrier species, namely, protons, the conductance of which can therefore be measured directly by impedance spectroscopy. ${ }^{11,12}$ Polymer electrolyte membranes (PEMs) based on perfluorinated sulfonic acids are in fact systems of crucial value for clean energy generation. PEMs are the key components of polymer electrolyte fuel cells, consuming hydrogen, the promising systems for mobile, portable, and residential applications. ${ }^{13}$

The PEM volume density can be determined through the polymer equivalent weight (the number of $\mathrm{SO}_{3}{ }^{-}$groups per gram of dry polymer) and the measured water uptake. Hence, values of single-proton mobility can be extracted, albeit not for the bulk aqueous phase but for the complex confined membrane environment. These results are of obvious interest for membrane science and technology, but they do not apply straightforwardly to proton mobility in pure water. Membrane proton mobility for high water uptake can, however, approach that of bulk water. It can be speculated that this is because most of the proton transport is then in the interior of the pores and because the protons have only limited ability to move along the surfaces of the water filled channels. ${ }^{14}$

Membrane proton conductance data have been obtained at ambient pressures in a temperature range from 170 to $310 \mathrm{~K}$. The data of Cappadonia et al. ${ }^{11,12}$ reveal two conductance regimes, with a change in activation energy between 225 and 260 K. Straight Arrhenius plots were obtained above $273 \mathrm{~K}$. The apparent activation energy decreases significantly on water uptake, reaching $0.1 \mathrm{eV}$ in water-saturated membranes. This is the value usually attributed to proton mobility in bulk water. Mean field theory ${ }^{14}$ explains this readily, but other explanations are needed if protons are localized more strongly near pore surfaces. All in all, the proton mobility in water should therefore not be assessed solely on the basis of membrane conductance even in a highly saturated state. There are other caveats with the simplest notion of excess proton mobility and the comparison with membrane proton conductance at water saturation. First, by taking the difference between the limiting conductance of, say, $\mathrm{HCl}$ and $\mathrm{NaCl}$, the anion contribution is canceled. The limiting conductance of $\mathrm{Na}^{+}$is also subtracted entirely, because this ion can move only by the classical mechanism. The limiting classical conductance of $\mathrm{H}_{3} \mathrm{O}^{+}$is, however, only partially canceled. Anticipating the discussion below, this is because $\mathrm{H}_{3} \mathrm{O}^{+}$exhibits classical motion only for part of the time. Such a difference is thus a direct measure of nonclassical proton conductance only when the classical, hydrodynamic mobility of hydrated $\mathrm{H}_{3} \mathrm{O}^{+}$is considerably smaller than the total proton mobility. A second observation relates to the contrasting temperature variation of the excess proton mobility in water (Figure 1) and proton mobility in saturated polymer electrolyte membranes. The former variation is strong and nonmonotonic in the high-temperature region. The latter is not only monotonic but also Arrhenius-like, at least above the freezing point of bulk water. At first glance, a plausible assumption would be that classical $\mathrm{H}_{3} \mathrm{O}^{+}$diffusion compensates for the decrease of the

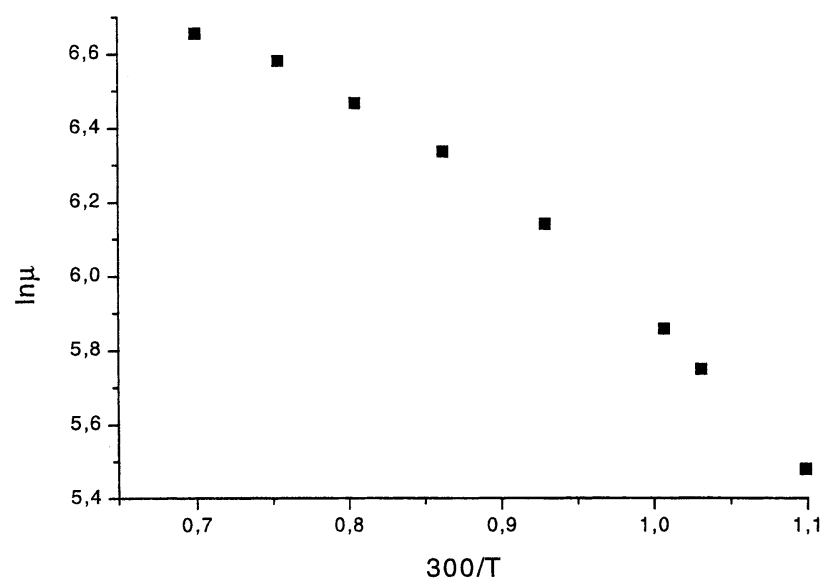

Figure 1. Experimental data for excess proton mobility. Taken from refs 2,6 , and 8 .

excess mobility at high temperatures. This is, however, inconsistent with the assumption that the classical $\mathrm{H}_{3} \mathrm{O}^{+}$contribution is small.

Theoretical attention to proton mobility in water began with Grotthus, ${ }^{15}$ at a time when the existence of the proton was not known, the chemical formula of water not settled, the notion of molecules was new, and little was known about stationary electricity. Modern landmarks were set by Bernal and Fowler, ${ }^{16}$ Eigen and de Mayer, ${ }^{17}$ Conway et al., ${ }^{18}$ and Zundel and Metzger. ${ }^{19}$ This was followed by more detailed molecular mechanisms and by analytical and computational models, for an overview, see ref 20 .

The fundamental importance of proton transfer (PT) in biology, ${ }^{21,22}$ and in the development of fuel cells, ${ }^{13,23,24}$ continues to press for a deeper understanding of PT mechanisms in hydrogen bonded systems. Recent molecular dynamics (MD) computer simulations have highlighted the nature of the elementary act of PT in water. ${ }^{28,30,31,25}$ They have provided new evidence for the crucial effect of the dynamics of solvation water molecules of the PT clusters. ${ }^{32}$

In one suggested mechanism, PT is initiated by the breaking of a hydrogen bond between the acceptor water molecule and a water molecule in its solvation shell. Hydrogen bond breaking ushers the acceptor molecule to a favorable configuration for accepting the proton, while the donor molecule forms a new hydrogen bond with a water molecule in its solvation shell. Regardless of details, together with classical hydronium ion motion, this mechanism will be denoted as the translocation of hydronium, or "mechanism I" in the following.

In this picture, the proton transport is viewed as occurring in a stepwise fashion: one PT after another, triggered by favorable nearest molecular environment and slow medium polarization fluctuations (involving hydrogen bond breaking and making). This mechanism in small hydration clusters was explored by Ando and Hynes ${ }^{26,27}$ via a combination of ab initio electronic structure calculations and Monte Carlo simulations. It was found that $\mathrm{H}_{2} \mathrm{O}$ plays a key role in the rearrangements of the first solvation shell water molecules around the $\mathrm{H}^{+}$donor and acceptor. These findings have provided relatively simple molecular patterns for solvent reorganization and outlined the role played by hydrogen bonds in PT.

Fully quantum Carr-Parinello MD simulations in larger aggregates of water molecules ${ }^{28}$ and infrared spectroscopy ${ }^{29}$ have shown, however, that the hydronium ion is not the only and possibly not even the most stable excess proton state. The proton probably spends equal or more time in the Zundel cluster 
$\mathrm{H}_{5} \mathrm{O}_{2}{ }^{+}$. The excess proton in this state is located approximately symmetrically between the oxygen atoms of two enclosing water molecules. The equilibrium $\mathrm{O}-\mathrm{O}$ distance is significantly shorter than the average $\mathrm{O}-\mathrm{O}$ distance in bulk water. As the proton potential energy profile depends crucially on the $\mathrm{O}-\mathrm{O}$ distance this profile reduces to a single or a shallow doublewell potential. This PT mechanism is different from hydronium translocation. The rate-determining step is likely to be transformation of a given $\mathrm{H}_{5} \mathrm{O}_{2}{ }^{+}$cluster into an adjacent cluster, a mechanism denoted as structural diffusion, according to

$$
\left(\mathrm{H}_{2} \mathrm{O} \cdots \mathrm{H}^{+} \cdot \cdots \mathrm{H}_{2} \mathrm{O}\right) \cdots \mathrm{H}_{2} \mathrm{O} \rightarrow \mathrm{H}_{2} \mathrm{O} \cdots\left(\mathrm{H}_{2} \mathrm{O} \cdot \cdot \mathrm{H}^{+} \cdot \cdots \mathrm{H}_{2} \mathrm{O}\right)
$$

Unlike hydronium translocation, structural Zundel complex diffusion, denoted in the following as mechanism II, involves displacement of either two or three protons. Mutual transformation between hydronium ions and Zundel complexes involves little charge transfer. The transformations can be characterized by forward and reverse rate constants, and both complexes can mediate PT.

Recent computer simulations of proton mobility in water have reached a powerful level. ${ }^{28}$ Importantly, simulations extend to quantum-mechanical proton dynamic features, where proton motion can be coupled to details of the molecular environmental dynamics. In view of the broad importance of PT and the need for facile comparison with experimental data, it would be interesting to have also a theoretical framework which rationalizes computational results and could be a handy tool for experimental data analysis. The present report focuses on such a framework, supported by new MD simulations. As in other recent MD simulations, these reduce the molecular proton conductivity mechanisms largely to single- and double-PT events in aqueous PT clusters with well-defined average structures. The computational results can therefore be interfaced both with classical views of dynamic water cluster structures and with contemporary theories of elementary charge-transfer processes in condensed media..$^{33,34,35}$ The latter is by no means confined to views based on displaced harmonic modes and weakly interacting donor and acceptor molecular entities. These theories extend in conceptually straightforward ways, for example, to anharmonic local mode dynamics and strong donor-acceptor interactions prevailing in the closely tied PT clusters in aqueous proton conduction.

There are several merits of such an approach. The powerful framework of contemporary charge transfer theories including proton tunneling, diabatic and adiabatic limits, kinetic deuterium isotope effects, etc., ${ }^{34,35}$ are integrated naturally in the analytical frames, and the resulting formalism is easy to use. The parameters of the elementary PT rate constants such as local and environmental reorganization Gibbs free energies are brought to rest firmly on the computational results. Their status is thus clearly beyond that of fitting parameters. By mutual thermal equilibrium among the clusters, the results extend naturally to the views of Agmon ${ }^{20}$ and the classical studies of Eucken and of Gierer and Wirtz. ${ }^{4,5,6,8}$ The models introduced phenomenologically in ref 8 and based on comprehensive experimental data are thus brought to rest on new and more sophisticated levels of computer simulations and PT rate formalism. Broad temperature ranges and nonmonotonic temperature variation of the proton conductivity can thus be included straightforwardly. All this offer, finally, a basis for future approaches to PT in composite heterogeneous environments such as proteins and proton conducting membranes.

\section{Diffusion Coefficient in a Three State Approximation}

The overall proton conductivity mechanism is regarded as being composed of hydrodynamic diffusion of bare or hydrated hydronium ion, single PT in a hydrated hydronium ion ("mechanism I", cf. above), and double or triple PT in a Zundel complex ("mechanism II"). All of the PT entities are dynamic entities and engaged in mutual labile equilibria. One approach to the stationary proton mobility or diffusion coefficient rests on evaluation of the quantity ${ }^{36}$

$$
D=\left.\lim \frac{\left\langle x^{2}(t)\right\rangle}{2 t}\right|_{t \rightarrow \infty}
$$

where $x$ is the displacement of the center of the excess charge from its position at the time origin. The long-time displacement does not depend on the type of the proton transferring entity ("bare" hydronium, hydrated hydronium, or Zundel complex) in the starting configuration because of the prevalence of mutual interconversions. The diffusion coefficient can be written as

$$
D=P_{1} W_{1} a_{1}^{2}+P_{2} W_{2} a_{2}^{2}+P_{3} W_{3} a_{3}^{2}
$$

where $P_{k}$ are the weights (relative concentrations) of the different PT clusters, while $W_{k}$ and $a_{k}$ are the corresponding transition probabilities per unit time and elementary PT distances of the clusters. $k=1,2$, and 3 stands for bare hydronium, hydrated hydronium, and Zundel complex, respectively. $P_{k}$ can be estimated from the equilibria between the charge transferring entities: $1 \Leftrightarrow 2,2 \Leftrightarrow 3$ assuming no direct transformation between states 1 and 3 . Introducing the rate constants $k_{12}, k_{23}$, $k_{21}$, and $k_{32}$ for the allowed transformations, we can write the equations of balance between the three states

$P_{1}+P_{2}+P_{3}=1 ; \quad k_{21} P_{2}-k_{12} P_{1}=0 ; \quad k_{23} P_{2}-k_{32} P_{3}=0$

Their solutions read

$$
\begin{gathered}
P_{1}=\frac{1}{1+K_{12}+K_{12} K_{23}} \\
P_{2}=\frac{1}{1+K_{21}+K_{23}} \\
P_{3}=\frac{1}{1+K_{21} K_{32}+K_{32}}
\end{gathered}
$$

where $K_{12}=k_{12} / k_{21}, K_{32}=k_{32} / k_{23}$, and $K_{23}=k_{23} / k_{32}$ are the corresponding equilibrium constants

$$
K_{12}=K_{21}^{-1}=A_{12} \mathrm{e}^{E_{12} / k_{\mathrm{B}} T}, \quad K_{23}=K_{32}^{-1}=A_{23} \mathrm{e}^{E_{23} / k_{\mathrm{B}} T}
$$

with the enthalpies $E$ and entropy factors $A$. Inserting eq 6 into eq 5 we obtain

$$
\begin{gathered}
P_{1}=\frac{1}{1+A_{12} \mathrm{e}^{E_{12} / k_{\mathrm{B}} T}+A_{12} A_{23} \mathrm{e}^{\left(E_{12}+E_{23}\right) / k_{\mathrm{B}} T}} \\
P_{2}=\frac{1}{1+\frac{1}{A_{12}} \mathrm{e}^{-\left(E_{12} / k_{\mathrm{B}} T\right)}+A_{23} \mathrm{e}^{E_{23} / k_{\mathrm{B}} T}} \\
P_{3}=\frac{1}{1+\frac{1}{A_{23}} \mathrm{e}^{-\left(E_{23} / k_{\mathrm{B}} T\right)}+\frac{1}{A_{12} A_{23}} \mathrm{e}^{-\left(E_{12}+E_{23}\right) / k_{\mathrm{B}} T}}
\end{gathered}
$$


Equations 3 and 7 give a general expression for the diffusion coefficient involving the transition probabilities, lengths of the hops, and equilibrium constants. The transition probabilities, $W_{k}$, are calculated for each mechanism separately. For each of the three mechanisms, $W_{k}$ can be written in the general form ${ }^{34,35}$

$$
W=N \frac{\omega_{\text {eff }}}{2 \pi} \kappa \exp \left(-\frac{G^{\neq}}{k_{\mathrm{B}} T}\right)
$$

with appropriate subscripts of $N, \omega_{\text {eff }}, \kappa$, and $G^{\neq} . N$ is a structural factor which depends on the number of possible PT directions, $\omega_{\text {eff }}$ is the effective vibrational frequency of all classical nuclear modes, $\kappa$ is the quantum mechanical transmission coefficient for the transferring proton, and $G^{\neq}$is the activation Gibbs free energy.

Equation 8 incorporates all of the features of each of the three charge-transfer mechanisms. $\omega_{\text {eff }}$ is thus a weighted frequency average of all of the nuclear modes displaced by thermally activated motion. In most cases $\omega_{\text {eff }}$ is close to $k_{\mathrm{B}} T / \hbar$ where $k_{\mathrm{B}}$ is Boltzmann's constant, $T$ is the temperature, and $2 \pi \hbar$ is Planck's constant. These modes are librational and translational motion of individual or clusters of water molecules and collective bulk polarization modes. $G^{\neq}$is the activation Gibbs free energy determined by the dynamics and structural reorganization of these modes. The proton dynamics is represented by the transmission coefficient $\kappa$, which reflects the quantum mechanical (tunneling) nature of the proton transition.

In the adiabatic limit of strong proton donor-acceptor interaction, $\kappa \rightarrow 1.34,35,37,38$ This limit prevails for excess aqueous proton conductivity. In the opposite, diabatic limit (with respect to proton/solvent mode separation), $\kappa \ll 1$. This limit prevails for weakly interacting proton donor and acceptor fragments. ${ }^{34,35}$ In the adiabatic limit, proton tunneling is still reflected in $G^{f}$ as a lower activation Gibbs free energy caused by the strong splitting of the Gibbs free energy surfaces in the crossing region between the reactant and product states. The splitting is also important for the kinetic deuterium isotope effect of excess proton conductivity in aqueous solution (section 7.4).

From the Debye-Einstein relationship, $\mu=e D / k_{\mathrm{B}} T$, the proton mobility, $\mu$, reads

$$
\mu=\frac{e}{k_{\mathrm{B}} T}\left[P_{1} W_{1} a_{1}^{2}+P_{2} W_{2} a_{2}{ }^{2}+P_{3} W_{3} a_{3}{ }^{2}\right]
$$

The excess mobility ${ }^{8}$ is then, finally

$$
\begin{aligned}
\mu_{\text {excess }}= & \frac{e}{k_{\mathrm{B}} T}\left[\left(P_{1}-1\right) W_{1} a_{1}^{2}+P_{2} W_{2} a_{2}^{2}+P_{3} W_{3} a_{3}^{2}\right]= \\
& \frac{e}{k_{\mathrm{B}} T}\left[P_{2}\left(W_{2} a_{2}^{2}-W_{1} a_{1}^{2}\right)+P_{3}\left(W_{3} a_{3}^{2}-W_{1} a_{1}^{2}\right)\right]
\end{aligned}
$$

In the following three sections, we consider the most important features of proton conduction in aqueous solution based on the molecular properties of the three "elementary" charge-transfer mechanisms. Direct support of the mechanisms by computer simulation will be addressed in section 6 .

\section{Classical Diffusion of the $\mathrm{H}_{3} \mathrm{O}^{+}$Ion}

The first PT mechanism in the proton conduction frame is diffusion of bare or hydrated $\mathrm{H}_{3} \mathrm{O}^{+}$as a whole. Computer simulations show that there is no such motion in pure form at room temperature, but if the excess proton were fixed on a given water molecule, $\mathrm{H}_{3} \mathrm{O}^{+}$motion would resemble that of a light cation. $^{25}$

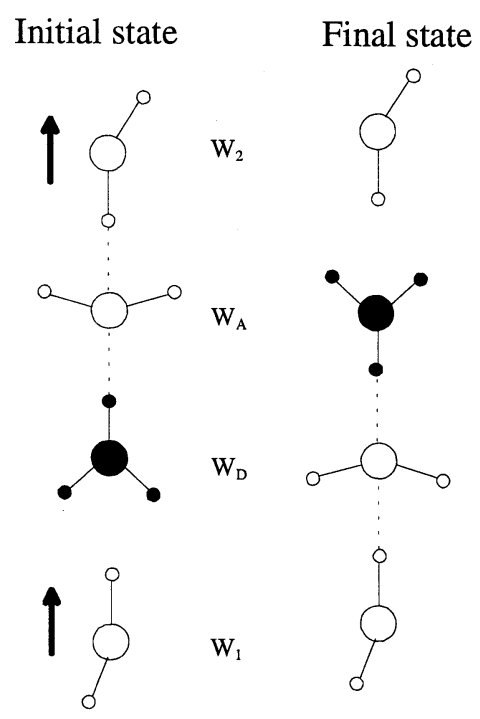

Figure 2. Structural diffusion of the hydronium ion. A scheme of proton transfer via proton hopping between two water molecules (only the key molecules in the process are shown; their hydrogen bonds with other water molecules are not displayed for clarity). In the initial state, an excess proton is bonded to the "donor" molecule $W_{\mathrm{D}}$ within the $\mathrm{H}_{3} \mathrm{O}^{+}$ ion (black). The acceptor molecule, $W_{\mathrm{A}}$, forms hydrogen bonds with $\mathrm{H}_{3} \mathrm{O}^{+}$and $W_{2}$. In the final state, an excess proton is bonded to the "acceptor" molecule $W_{\mathrm{A}}$ within the new $\mathrm{H}_{3} \mathrm{O}^{+}$ion (black); the molecule $W_{\mathrm{D}}$ forms hydrogen bonds with the new $\mathrm{H}_{3} \mathrm{O}^{+}$and $W_{2}$.

Microscopic mechanisms of ion motion in polar liquids are, however, not unambiguously determined. At large time scales, the ion (with its solvation shell depending on the strength of solvation) moves as a Stokes sphere, whereas at short time scales, its motion is strongly correlated with orientational dipolar solvent fluctuations. The latter resemble hopping type motion between neighboring localizations upon reorientational fluctuation of the dipolar environment. ${ }^{39}$ This determines the Arrhenius form of the temperature dependence of the diffusion coefficient.

Classical (hydrated) $\mathrm{H}_{3} \mathrm{O}^{+}$diffusion can therefore still be regarded as a considerable contribution, provided that $\mathrm{H}_{3} \mathrm{O}^{+}$is viewed as a dynamic molecular entity. This contribution is more prominent at higher temperatures where the highly organized molecular cluster structures which control the PT mechanisms are increasingly dissipated. Equation 8 is appropriate for parametrization of the transition probability based on this transfer mechanism, with $\kappa=1, N=1 / 3, \omega_{\text {eff }} \approx 10^{13} \mathrm{~s}^{-1}$. The activation free energy, according to simulations, is in the region $0.05-0.09 \mathrm{eV}$.

\section{Proton Hops between Two Water Molecules}

4.1. Model. PT from a hydronium ion to the nearest water molecule can be viewed as intramolecular chemical PT in a reaction complex consisting of two water molecules and the excess proton (Figure 2). In the initial state, the proton is chemically bound to the donor molecule $\left(\mathrm{H}_{2} \mathrm{O}\right)_{D}$ forming the hydronium ion $\mathrm{H}_{3} \mathrm{O}^{+}$hydrogen bonded to the acceptor molecule $\left(\mathrm{H}_{2} \mathrm{O}\right)_{\mathrm{A}}$. Two other neighboring molecules $\left(\mathrm{H}_{2} \mathrm{O}\right)_{\mathrm{w} 1}$ and $\left(\mathrm{H}_{2} \mathrm{O}\right)_{\mathrm{w} 2}$ are crucial. In the initial state $\left(\mathrm{H}_{2} \mathrm{O}\right)_{\mathrm{w} 2}$ forms a hydrogen bond with the acceptor molecule $\left(\mathrm{H}_{2} \mathrm{O}\right)_{\mathrm{A}}$, whereas $\left(\mathrm{H}_{2} \mathrm{O}\right)_{\mathrm{w} 1}$ is close to the hydronium ion but without establishing a hydrogen bond.

The mechanism consists of synchronous motion of $\left(\mathrm{H}_{2} \mathrm{O}\right)_{\mathrm{w}}$ toward the donor molecule $\left(\mathrm{H}_{2} \mathrm{O}\right)_{\mathrm{D}}$ and of $\left(\mathrm{H}_{2} \mathrm{O}\right)_{\mathrm{w} 2}$ away from the acceptor molecule $\left(\mathrm{H}_{2} \mathrm{O}\right)_{\mathrm{A}}$. This leads to a shift of the proton from $\left(\mathrm{H}_{2} \mathrm{O}\right)_{D}$ to $\left(\mathrm{H}_{2} \mathrm{O}\right)_{A}$, formation of the hydrogen bond $\left(\mathrm{H}_{2} \mathrm{O}\right)_{\mathrm{w} 1} \cdots\left(\mathrm{H}_{2} \mathrm{O}\right)_{\mathrm{D}}$, and breaking of the hydrogen bond 
$\left(\mathrm{H}_{2} \mathrm{O}\right)_{\mathrm{A}} \cdots\left(\mathrm{H}_{2} \mathrm{O}\right)_{\mathrm{w} 2}$ (Figure 2). PT is facilitated also by a fluctuational decrease of the distance between the oxygen atoms of the donor and acceptor molecules. The formal scheme reads

$$
\begin{aligned}
&\left(\mathrm{H}_{2} \mathrm{O}\right)_{\mathrm{w} 1}+\left(\mathrm{H}_{2} \mathrm{O}\right)_{\mathrm{D}}-\mathrm{H}^{+} \cdots\left(\mathrm{H}_{2} \mathrm{O}\right)_{\mathrm{A}} \cdots\left(\mathrm{H}_{2} \mathrm{O}\right)_{\mathrm{w} 2} \Longrightarrow \\
&\left(\mathrm{H}_{2} \mathrm{O}\right)_{\mathrm{w} 1} \cdots\left(\mathrm{H}_{2} \mathrm{O}\right)_{\mathrm{D}} \cdots \mathrm{H}^{+}-\left(\mathrm{H}_{2} \mathrm{O}\right)_{\mathrm{A}}+\left(\mathrm{H}_{2} \mathrm{O}\right)_{\mathrm{w} 2}
\end{aligned}
$$

with dots denoting hydrogen bonds. All other water dipoles affect the transition mainly through electrostatic interactions with the charge distribution of the reaction complex and assume the role of an effective medium.

4.2. "Reactive Modes" and Free Energy Surfaces. The general form in eq 8 remains valid for the single-PT model in Figure 2, but attention to more kinematic details than for hydrodynamic $\mathrm{H}_{3} \mathrm{O}^{+}$diffusion is required. Chemical PT theory ${ }^{34,35}$ is the most suitable and convenient frame for this consideration, starting with the potential Gibbs free energy surfaces of the system in the reactants' and products' states. The Gibbs free energy surfaces can be constructed either from the diabatic reactants' and products' surfaces or from the unified system potential surface in the ground state of all electronic and vibrational high-frequency (proton) system parts.

The notion of potential Gibbs free energy surfaces is rooted in two observations. One is that the kinetic parameters of analytical rate theory of condensed phase chemical processes, particularly the activation free energy, are determined by thermal fluctuations along central reactive modes. This notion implies that the contributions of all other nuclear modes, for example bulk bath modes, are statistically averaged. Motion along the reactive modes therefore becomes determined by the system free energy and the potential surfaces by the reactive modes, free energy surfaces. An implication of this concept is that the parameters of suitable model potentials chosen to represent the reactive modes have a temperature coefficient. The second observation is that the environmental components of the potential surfaces are represented by the inertial polarization fluctuations. These are macroscopic quantities and determined by the associated polarization free energy. In dielectric continuum theory, the latter involves, for example, the dielectric permittivity of the solvent, which clearly depends on the temperature.

The potential surfaces are the basis for calculation of the activation Gibbs free energy and are spanned by all the reactive nuclear coordinates ("modes"), i.e., those displaced or distorted in the PT event.

In the model shown in Figure 2, the reactive nuclear modes include (1) the distance between the oxygen atoms, $R$, in the donor and acceptor molecules $\left(\mathrm{H}_{2} \mathrm{O}\right)_{\mathrm{D}}$ and $\left(\mathrm{H}_{2} \mathrm{O}\right)_{\mathrm{A}}$, (2) the coordinate of the transferable proton $r_{\mathrm{p}}$ along the straight line connecting the two oxygen atoms of $\left(\mathrm{H}_{2} \mathrm{O}\right)_{D}$ and $\left(\mathrm{H}_{2} \mathrm{O}\right)_{A}$, (3) the distance $\mathrm{x}$ between $\left(\mathrm{H}_{2} \mathrm{O}\right)_{\mathrm{w} 1}$ and $\left(\mathrm{H}_{2} \mathrm{O}\right)_{\mathrm{D}},(4)$ the distance $y$ between $\left(\mathrm{H}_{2} \mathrm{O}\right)_{\mathrm{w} 2}$ and $\left(\mathrm{H}_{2} \mathrm{O}\right)_{\mathrm{A}}$, and (5) the set of coordinates $\left\{q_{k}\right\}$ of the effective oscillators describing the bulk polarization of the medium outside the reaction complex.

It is assumed that the other protons in the $\left(\mathrm{H}_{2} \mathrm{O}\right)_{\mathrm{D}}$ and $\left(\mathrm{H}_{2} \mathrm{O}\right)_{\mathrm{A}}$ molecules as well as the electrons follow adiabatically the instant reactive mode configurations. Their coordinates therefore do not appear explicitly. It is most convenient to start from diabatic free energy surfaces describing the separate initial $\left(U_{\mathrm{i}}\right)$ and final $\left(U_{\mathrm{f}}\right)$ states. These represent, respectively, the separate reactants' and products' states exclusive of the "exchange" interaction between $\left(\mathrm{H}_{2} \mathrm{O}\right)_{D}$ and $\left(\mathrm{H}_{2} \mathrm{O}\right)_{A}$. The separate reactants' and products' states are subsequently used to construct the lower adiabatic Gibbs free energy surface, obtained after inclusion of the exchange interaction. ${ }^{34,35}$

The interaction between the donor and acceptor molecules is strong, i.e., a considerable fraction of an eV (e.g., refs 37, 38 , and 40-44). From available criteria, ${ }^{33-35}$ the PT process is therefore well within the fully adiabatic limit. The preexponential form in eq 8 therefore accords with the parameter values $N=1, \kappa=1$, and $\omega_{\text {eff }} \approx k_{\mathrm{B}} T / \hbar \approx 10^{13} \mathrm{~s}^{-1}$. The motion along all reactive nuclear coordinates $\left(x, y, R\right.$, and $\left.\left\{q_{k}\right\}\right)$ except those of the protons $\left(r_{\mathrm{p}}\right)$ is, moreover, classical. The motion of the protons can be separated from the motion of other nuclei with the use of the Born-Oppenheimer approximation. ${ }^{45}$ The transition path crosses the saddle point on the reduced adiabatic free energy surface for the proton vibrational ground state, i.e., the surface spanned solely by the coordinates of heavy nuclei. ${ }^{34,35}$ In the Born-Oppenheimer approximation, the proton energies, $E_{\mathrm{pi}}\left(x, y, R,\left\{q_{k}\right\}\right)$ and $E_{\mathrm{pf}}\left(x, y, R,\left\{q_{k}\right\}\right)$, and wave functions, $\chi_{\mathrm{i}}\left(r_{\mathrm{p}} ; x, y, R,\left\{q_{k}\right\}\right)$ and $\chi_{\mathrm{f}}\left(r_{\mathrm{p}} ; x, y, R,\left\{q_{k}\right\}\right)$ of the initial (i) and final (f) states are calculated from the Schrödinger equation at fixed values of the classical coordinates.

The reduced free energy surface of the initial, or reactant, state $U_{\mathrm{i}}^{\mathrm{r}}\left(x, y, R,\left\{q_{k}\right\}\right)$ is

$U_{\mathrm{i}}^{\mathrm{r}}\left(x, y, R,\left\{q_{k}\right\}\right)=E_{\mathrm{pi}}\left(x, y,\left\{q_{k}\right\} ; R\right)+W_{\mathrm{i}}^{\mathrm{s}}\left(\left\{q_{k}\right\} ; x, y\right)+V_{\mathrm{i}}(x, y, R)$

$E_{\mathrm{pi}}$ is the proton ground-state eigenvalue in the hydronium ion, counted from the minimum energy of the proton potential well, which is included in the other potential terms in eq 12 , see below. $W_{\mathrm{i}}^{\mathrm{s}}$ is the free energy of the outer-sphere water polarization. ${ }^{33-35} V_{\mathrm{i}}$ couples the three modes $x, y$, and $R$, a broadly valid form being

$$
V_{\mathrm{i}}(x, y, R) \approx V^{\mathrm{i}}(x, R)+u_{2}\left(y-y_{0 \mathrm{i}}(x, R)\right)
$$

The first term in eq 13 includes the interaction free energy of the water molecule $\left(\mathrm{H}_{2} \mathrm{O}\right)_{\mathrm{w} 1}$ with the oxygen atom of the donor molecule and the $\mathrm{O}-\mathrm{O}$ interaction between $\left(\mathrm{H}_{2} \mathrm{O}\right)_{D}$ and $\left(\mathrm{H}_{2} \mathrm{O}\right)_{\mathrm{A}}$ at equilibrium, $y=y_{0 \mathrm{i}}(x, R)$, of the water molecule $\left(\mathrm{H}_{2} \mathrm{O}\right)_{\mathrm{w} 2}$. The second term describes the vibrational potential of $\left(\mathrm{H}_{2} \mathrm{O}\right)_{\mathrm{w} 2}$.

The free energy surface of the final, or product, state is, similarly

$$
\begin{gathered}
U_{\mathrm{f}}^{\mathrm{r}}\left(x, y, R,\left\{q_{k}\right\}\right)=V_{\mathrm{f}}(x, y, R)+E_{\mathrm{pf}}\left(x, y,\left\{q_{k}\right\} ; R\right)+W_{\mathrm{f}}^{\mathrm{s}}\left(\left\{q_{k}\right\} ; x, y\right) \\
V_{\mathrm{f}}(x, y, R) \approx V^{\mathrm{f}}(y, R)+u_{1}\left(x-x_{0 \mathrm{f}}(y, R)\right)
\end{gathered}
$$

The ground-state adiabatic free energy surface is constructed from $U_{\mathrm{i}}^{\mathrm{r}}$ and $U_{\mathrm{f}}^{\mathrm{r}} 34,35$

$$
U=\frac{1}{2}\left[U_{\mathrm{i}}^{\mathrm{r}}+U_{\mathrm{f}}^{\mathrm{r}}-\sqrt{\left(U_{\mathrm{i}}^{\mathrm{r}}-U_{\mathrm{f}}^{\mathrm{r}}\right)^{2}+\left[\Delta E_{\mathrm{h}}(x, y, R)\right]^{2}}\right]
$$

where $\Delta E_{\mathrm{h}}(x, y, R)$ is the quantum mechanical splitting of the proton energy levels in the proton double-well potential between $\left(\mathrm{H}_{2} \mathrm{O}\right)_{D}$ and $\left(\mathrm{H}_{2} \mathrm{O}\right)_{\mathrm{A}} \cdot{ }^{34,35,45}$

4.3. Activation Free Energy. The adiabatic transition from the reactants' to the products' equilibrium configurations is along the modes $x, y, R$, and $\left\{q_{k}\right\}$ on the proton vibrational ground- 
state adiabatic free energy surface. The activation Gibbs free energy is determined by the saddle point on this surface

$$
\begin{array}{r}
G^{\not \neq}=\frac{1}{4} E_{\mathrm{r}}\left(x_{\mathrm{s}}, y_{\mathrm{s}}, R_{\mathrm{s}}\right)+V^{\dot{\mathrm{i}}}\left(x_{\mathrm{s}}, R_{\mathrm{s}}\right)-V^{\mathrm{i}}\left(x_{0}, R_{0}\right)+ \\
u_{2}\left(y_{\mathrm{s}}-y_{0 \mathrm{i}}\left(x_{\mathrm{s}}, R_{\mathrm{s}}\right)\right)-\frac{1}{2} \Delta E_{\mathrm{h}}\left(x_{\mathrm{s}}, y_{\mathrm{s}}, R_{\mathrm{s}}\right)
\end{array}
$$

where the subscript $s$ denotes the coordinate values at the saddle point. The equations for the saddle point are given in Appendix A. $E_{\mathrm{r}}$ is the solvent reorganization energy.

Equation 17 is general for the mechanism in Figure 2. To utilize it, we need to specify the potential terms in this equation. This involves two approximations. One is the decoupling of $x$ and $R$ in the potential $V^{i}(x, R)$. This term can be separated into a sum of the interaction of the nonbonded water molecule with the hydronium ion and the $\mathrm{O}-\mathrm{O}$ interaction between the donor and acceptor molecules:

$$
V^{\mathrm{i}}(x, R) \approx V_{\mathrm{w}_{1}-\mathrm{O}}^{\mathrm{i}}(x)+V_{\mathrm{O}-\mathrm{O}}(R)
$$

The second approximation concerns the particular form of $u_{2}$ and $V_{\mathrm{w}_{1}-\mathrm{O}}^{\mathrm{i}}(x)$. The potential of the hydrogen bond, $u_{2}$, can be approximated by a Morse function, and the repulsive branch of the interaction of the nonbonded water molecule with the hydronium ion, $V_{\mathrm{w}_{1}-\mathrm{O}}^{\mathrm{i}}(x)$, can be approximated by an exponential function (Appendix B). A minimized parameter set (Appendix B) then gives for the activation barrier

$$
\begin{array}{r}
G^{\neq}=\frac{1}{4} E_{\mathrm{r}}\left(x_{\mathrm{s}}, y_{\mathrm{s}}, R_{\mathrm{s}}\right)+\frac{1}{2} D_{\mathrm{HB}}-V_{\mathrm{w}_{1}-\mathrm{O}}^{\mathrm{i}}\left(x_{0}\right)+V_{\mathrm{O}-\mathrm{O}}\left(R_{\mathrm{s}}\right)- \\
V_{\mathrm{O}-\mathrm{O}}\left(R_{0}\right)-\frac{1}{2} \Delta E_{\mathrm{h}}\left(x_{\mathrm{s}}, y_{\mathrm{s}}, R_{\mathrm{s}}\right)
\end{array}
$$

where $D_{\mathrm{HB}}$ is the dissociation energy of the hydrogen bond.

\section{Proton Mobility via Zundel-Like Complexes}

5.1. Model and Reactive Modes. Zundel complexes constitute the initial (reactants') and final (products') PT states in mechanism II. In each complex, the proton is located approximately in the middle between two water molecules with considerably shorter $\mathrm{O}-\mathrm{O}$ distance than the $\mathrm{O}-\mathrm{O}$ distances in bulk water. In the PT scenario, a water molecular configurational fluctuation first increases the $\mathrm{O}-\mathrm{O}$ distance, $R_{12}$, in the initial Zundel complex, whereas the distance $R_{23}$ between one of the oxygen atoms of this complex and the oxygen atom of the nearest neighbor molecule decreases (Figure 3). This results in a synchronous shift of two protons, leading to the disappearance of the initial Zundel complex and formation of a new one. Water molecules of the second solvation shell of the Zundel complex are also important in the transition, but we restrict ourselves to two reactive modes $R_{12}$ and $R_{23}$ and include all other modes in the "outer-sphere" medium polarization. This view is the basis for construction of the potential Gibbs free energy surfaces and rate constants.

5.2. Gibbs Free Energy Surfaces. We denote the oxygen atoms of the three water molecules directly involved in the transition as $\mathrm{O}_{1}, \mathrm{O}_{2}$, and $\mathrm{O}_{3}$ (Figure 3). The reduced free energy surface of the initial (reactants') state is

$$
\begin{array}{r}
U_{\mathrm{i}}^{\mathrm{r}}\left(R_{12}, R_{23},\left\{q_{k}\right\}\right)=V_{23}^{\mathrm{i}}\left(R_{23}\right)+u_{12}\left(R_{12}-R_{12}^{0 \mathrm{i}}\left(R_{23}\right)\right)+ \\
W_{\mathrm{i}}^{\mathrm{s}}\left(\left\{q_{k}\right\} ; R_{12}, R_{23}\right)
\end{array}
$$
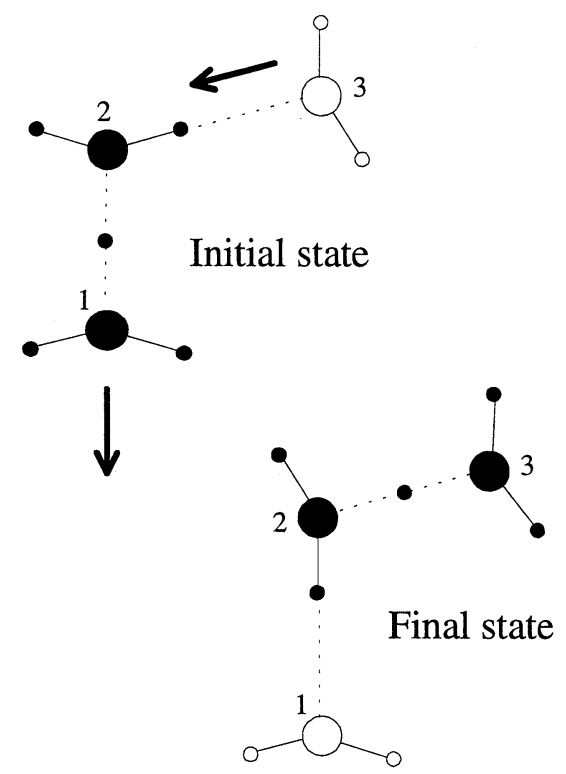

Figure 3. Structural diffusion of the Zundel complex. A scheme of proton transfer via the transition of two protons in a molecular triad. (Only the key molecules in the process are shown; their hydrogen bonds with other molecules are not displayed). The excess proton is initially localized in the initial Zundel complex (black). The nearest neighbor water molecule which forms a hydrogen bond with oxygen (2) is shown. In the final state, this proton is bonded to oxygen (2), while another proton is now located inside the new Zundel complex (black).

The first term represents the interaction of the Zundel complex with the nearest water molecule of the solvation shell, the second term represents the $\mathrm{O}_{1}-\mathrm{O}_{2}$ vibrational potential in the Zundel complex, and the last term represents the free energy of the inertial bulk medium polarization.

The reduced free energy surface of the final (products') state has a similar form

$$
\begin{array}{r}
U_{\mathrm{f}}^{\mathrm{r}}\left(R_{12}, R_{23},\left\{q_{k}\right\}\right)=V_{12}^{\mathrm{f}}\left(R_{12}\right)+u_{23}\left(R_{23}-R_{23}^{0 \mathrm{f}}\left(R_{12}\right)\right)+ \\
W_{\mathrm{f}}^{\mathrm{s}}\left(\left\{q_{k}\right\} ; R_{12}, R_{23}\right)
\end{array}
$$

The first term is again the interaction with the nearest water molecule of the solvation shell and the second term the $\mathrm{O}_{2}-\mathrm{O}_{3}$ potential, in the new Zundel complex.

The adiabatic free energy surface is obtained from an equation similar to eq 16

$$
U=\frac{1}{2}\left[U_{\mathrm{i}}^{\mathrm{r}}+U_{\mathrm{f}}^{\mathrm{r}}-\sqrt{\left(U_{\mathrm{i}}^{\mathrm{r}}-U_{\mathrm{f}}^{\mathrm{r}}\right)^{2}+\left[\Delta E_{\mathrm{Z}}\left(R_{12}, R_{23}\right)\right]^{2}}\right]
$$

where $\Delta E_{\mathrm{Z}}\left(R_{12}, R_{23}\right)$ is the resonance splitting of the two proton vibrational energy levels in the two-dimensional potential spanned by the coordinates of both protons.

5.3. Activation Gibbs Free Energy. The preexponential factor accords with $N=4 / 3, \kappa=1$, and $\omega_{\text {eff }} \approx k_{\mathrm{B}} T / \hbar \approx 10^{13}$ $\mathrm{s}^{-1}$. The activation barrier is calculated from

$$
\begin{array}{r}
G^{\neq}=\frac{1}{4} E_{\mathrm{r}}\left(R_{12}^{\mathrm{s}}, R_{23}^{\mathrm{s}}\right)+V_{23}^{\mathrm{i}}\left(R_{23}^{\mathrm{s}}\right)+u_{12}\left(R_{12}^{\mathrm{s}}-R_{12}^{0 \mathrm{i}}\left(R_{23}^{\mathrm{s}}\right)\right)- \\
\frac{1}{2} \Delta E_{\mathrm{Z}}\left(R_{12}^{\mathrm{s}}, R_{23}^{\mathrm{s}}\right)
\end{array}
$$

"s" refers again to the saddle point of the ground-state adiabatic free energy surface.

Morse-exponential potentials for the reactive modes and a factorial exponential dependence of the resonance energy splitting on the reactive mode coordinates (Appendix C) are 
appropriate specific approximations. A minimized parameter set (Appendix C) gives for the activation barrier

$$
G^{\neq}=\frac{1}{4} E_{\mathrm{r}}+\frac{1}{2} D_{\mathrm{Z}}-\frac{\Delta E_{0}}{8\left(1-\frac{\Delta E_{0}}{4 D_{\mathrm{Z}}}\right)}
$$

where $D_{\mathrm{Z}}$ is the dissociation energy of the Zundel complex and $\Delta E_{0}$ is the resonance energy splitting at equilibrium of the reactive coordinates.

We note that the saddle point is also determined by the coordinate dependence of the resonance energy splitting. This affects in turn the activation free energy (see Appendix C).

\section{MD Simulations}

We have illuminated the views above by extending and reanalyzing molecular dynamics (MD) simulations. This analysis provides, first and foremost, an insight into the mechanistic details of the diffusion process. The MD data provide, moreover, a basis for the interpretation of the experiments of Eucken ${ }^{4}$ and the analysis of Gierer and Wiertz, ${ }^{8}$ and identifies the nature of the dominating clusters that constitute the environment of intramolecular single- and double-PT.

A simple electronically polarizable two-state extended valence bond (EVB) model developed in ref 25 was used. This is currently the only model that allows the simulation of proton mobility in a large water "bath" at high proton concentrations. The price paid for that is the essential simplification of the PT algorithm. The essential elements of this simplification are as follows.

(i) The charge switching function which designates when PT takes place does not depend explicitly on the solvent environment. This may not be a serious limitation, because the conditions of transfer are entirely and self-consistently determined by the short- and long-range medium fluctuations, which may still somewhat affect the preexponential factor in proton mobility.

(ii) One proton is transferred at a time; that is, concerted pathways involving more than one proton are ignored from the outset, although the stepwise PTs may succeed each other so fast (as seen in computer animations of the simulation trajectories) that they would look almost like concerted. Eliminating this drawback could considerably affect the preexponential factor of proton mobility. Indeed, even if concerted pathways were rare, they could contribute nonnegligibly to mobility, because of the square of the distance factor in eq 3 . These shortcomings may become more substantial if the model is applied to proton transport in very narrow pores of proton conducting membranes, where the expected single-pile effects ${ }^{46}$ rest exactly on concerted proton-proton motion.

(iii) The "quantum" proton motion is simulated by classical mechanics. This shortcoming may not be serious, because we do not envisage under-barrier tunneling for the proton in the strongly adiabatic mechanism of PT, but this effect could be reflected in the KIE (see the discussion in section 7.4)

In ref 25 , the temperature dependence of the proton diffusion coefficient was found to be Arrhenius-like with an activation energy of $0.11 \mathrm{eV}$, close to the experimental estimates. However, there are presumably, the caveats of the model just listed that make the preexponential factor of proton mobility two times lower than it should be and the water diffusion too lively. Changing the model in such a way that the water diffusion is

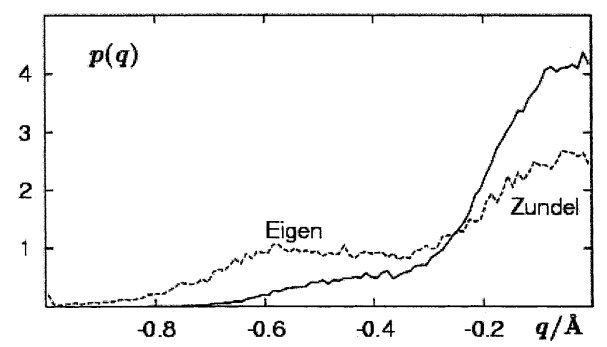

Figure 4. Distribution of the proton-transfer coordinate $q$. Calculated from simulations of one proton dissolved in 100 water molecules at $300 \mathrm{~K}$ (full line) and at $500 \mathrm{~K}$ (dashed). $q$ is the defined as the difference between the shorter and the longer of the two bridging $\mathrm{OH}$ distances in a Zundel complex. The $q$-regions corresponding to Zundel and Eigen complexes are indicated.

reduced to the experimentally observed value would thus also decrease the prexponential factor of the proton diffusion coefficient.

There is, however, hardly any choice. At low proton concentrations and smaller number of water molecules and also for shorter simulation time, several other more sophisticated choices, involving a larger number of valence bond states, are possible. ${ }^{47-49}$ However, to evaluate proton mobility (via the excess charge diffusion coefficient), we need longer simulations and larger systems. We therefore, here adopt the model of ref 25 with the expectation that it might be as suitable for a comparative analysis as the more sophisticated models. This is a strong conjecture, which needs verification in future largescale simulations.

We performed constant volume simulations of the transport of a single proton dissolved in 100 water molecules at temperatures of $300,330,360,400,500$, and $600 \mathrm{~K}$. The density in all runs is the density of bulk water at $298 \mathrm{~K}$ and ambient pressure. All interaction parameters and simulation conditions are identical to those in ref 25 . Below, we analyze the dynamics in terms of the simple overall jump pattern discussed in section 2. To this end, we study the temperature dependence of the distribution of proton states and then proceed to the calculation of the jump rates as a function of temperature. Finally, we relate the diffusion and jump activation barriers to structural properties.

6.1. Distribution of Proton-Transfer Coordinate. Following ref 25, we define the proton-transfer coordinate $q$ as the difference between the shorter and the longer $\mathrm{OH}$ distance in a Zundel complex. $q=0$ corresponds to the symmetric Zundel ion, and large negative values correspond to free or hydrated hydronium ions which we denote as Eigen complexes. Figure 4 shows the distribution of this coordinate at 300 and $500 \mathrm{~K}$. At room temperature, the model displays preference for the symmetric Zundel ion. This is supported by more general EVB models ${ }^{30,31}$ and by ab initio MD simulations. ${ }^{28}$ Although a distinction between Eigen and Zundel species is ambiguous because of the width of the distribution, it is clear that the equilibrium is shifted toward the Eigen complex with increasing temperature. This accords with the findings of Gierer and Wirtz, and of Eucken.

6.2. Proton Jumps. The Walbran and Kornyshev ${ }^{25}$ model describes proton transport through a sequence of interconversions between Zundel and Eigen complexes. The MD analysis shows that these interconversions are frequent. This can also be inferred from the shallow proton coordinate distribution in Figure 4 which shows no pronounced minima. Consequently, interconversions between Zundel and Eigen complexes with one of the two oxygens of the Zundel ion at the center of the Eigen complex look more like equilibrium fluctuations than quasi- 


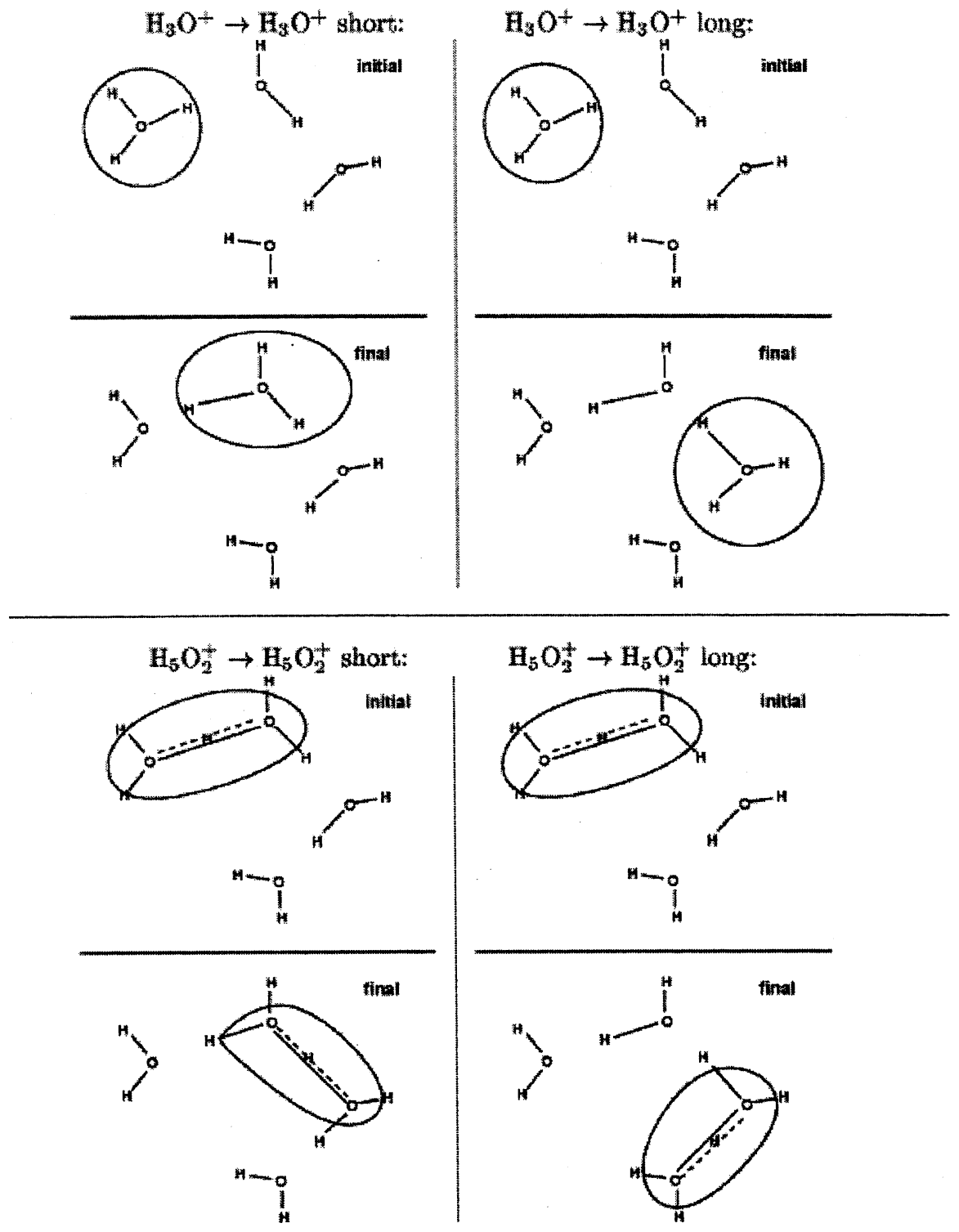

Figure 5. Sketches of various proton-transfer scenarios. The top half shows possible proton jumps between two Eigen complexes (or hydronium ions). The bottom half shows possible proton jumps between two Zundel complexes. Short jumps are on the left, long jumps on the right.

chemical jump processes. Other definitions for a PT step involve the transformation from a given Eigen complex to a neighboring one (with a different oxygen atom at the center) or the conversion of a given Zundel complex to another Zundel complex, where the second complex may or may not share an oxygen atom with the first one.

Four different PT scenarios involving short and long PT jumps are summarized in Figure 5. In the short Eigen-Eigen jumps, the center of the Eigen complex moves from the initial oxygen to one of its neighbors. In the long jump, the final Eigen complex is centered around an oxygen atom which was not part of the initial Eigen complex. This oxygen atom is most likely a second neighbor of the initial center. In a similar way we can define Zundel-Zundel jumps: a short jump corresponds to the transformation of the initial to the final Zundel ion, which still contains one of the two oxygen atoms of the initial Zundel ion. In the long jump, none of the two oxygen atoms of the initial Zundel complex are part of the final Zundel complex. We analyze the MD trajectories separately for each of the scenarios.

We define the time of formation of a particular Eigen ion as follows. That will be the first time when the proton-transfer coordinate $q$ becomes smaller than $-0.45 \AA$ with a particular oxygen at the center of the complex. We then wait, until another Eigen ion is formed (again with $q<-0.45 \AA$ ). This is called the transition time for the short or the long Eigen-Eigen jump, depending on whether the new Eigen center was part of the initial Eigen complex.

Similarly, we define the time of formation of a particular Zundel ion as the first time that the two particular oxygen atoms in a Zundel complex assume a value of $q$ larger than $-0.05 \AA$. Again we wait until a new Zundel complex (containing at least one new oxygen atom) is formed (with $q>-0.05 \AA$ ). We call this the transition time for the short or the long Zundel-Zundel jump, depending on whether the final Zundel complex does or does not share an oxygen atom with the initial one.

We collect the statistics over these events (typically several hundred or thousand for a simulation lasting three to four nanoseconds) and calculate the average transition rate. Figure 6 shows the average rates in an Arrhenius plot as a function of reciprocal temperature. The full symbols are for the long jump scenarios, and the open symbols are for the short jump scenarios. The two lines give the approximate activation energies of 0.04 


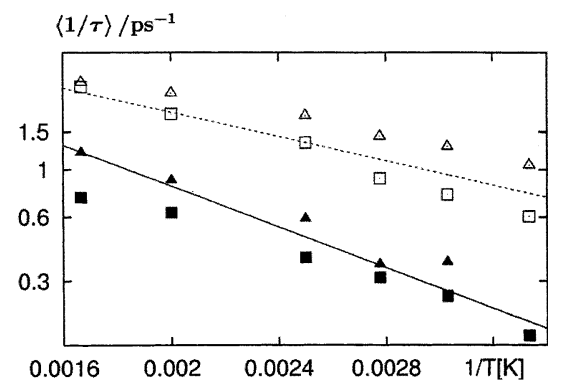

Figure 6. Arrhenius plot of the temperature dependence of the mean hopping times for the four different proton jumps sketched in Figure 5. Full symbols are for long jumps, and open symbols are for short jumps. Triangles are for Eigen-Eigen jumps, and squares are for Zundel-Zundel jumps. The lines correspond to activation energies of 0.04 (dashed) and $0.07 \mathrm{eV}$, respectively. Average times are calculated as the inverse of the average hopping rate. The activation energy for the long jumps is closer to the activation energy for the calculated diffusion step, thus making proton jumps between second-nearest neighbors more likely to be rate-determining for proton diffusion than the shorter jumps.

$\mathrm{eV}$ for the short jumps and $0.07 \mathrm{eV}$ for the long jumps. For comparison, the diffusion activation energy calculated in ref 25 is $0.11 \mathrm{eV}$. The data suggest that proton jumps involving only nearest neighbors are not rate-determining. Instead proton jumps to second nearest neighbors show a temperature dependence significantly closer to the one observed for the diffusion coefficient, indicating that they are related to the rate determining step for proton transport.

We also note that depending on temperature the number of short jumps in a given time is two to four times higher than the number of long jumps. Although the shorter jumps thus occur more frequently and with lower activation energy, their successive directions are anti-correlated in such a way that the probability for the next jump to be in the opposite direction of the previous one is significantly enhanced. (The non-Marcovian, anticorrelated character of the sequence of short jumps may, however, be an exagerration of the Walbran-Kornyshev model, which essentially results in a lowered preexponential factor of proton mobility.)

6.3. Structural Correlation Functions. In a symmetrical Zundel ion, the average oxygen-oxygen separation is about $2.4 \AA$; in an Eigen complex, the average oxygen-oxygen separation between the central oxygen atom and the others is $2.56 \AA$. The nearest-neighbor distance between two water molecules in the bulk is $\approx 2.85 \AA$. A proton jump between two positions in the liquid is thus associated with the relaxation of oxygen-oxygen distances. To relate the proton jump dynamics to such structural features, we have calculated distribution functions for the joint occurrence of two oxygen-oxygen distances. The oxygen-oxygen distance within the central Zundel complex (or, if the state of the proton is Eigen-like, the distance between the central water and its closest neighbor in the Eigen complex) is called $r_{12}$. The distances between any of these two oxygen atoms and the oxygen atoms of their nearest neighbors are called $r_{23}$. Finally, the distances between the nearest neighbors and their nearest neighbors (excluding the original two oxygen atoms) are termed $r_{34}$ (see Figure 7).

We calculate the probabilities $p\left(r_{12}, r_{23}\right)$ and $p\left(r_{12}, r_{34}\right)$ by monitoring the appropriate distance pairs during the simulation. The distributions have been symmetrized according to $\tilde{p}\left(r_{12}, r_{23}\right)=\max \left[p\left(r_{12}, r_{23}\right), p\left(r_{23}, r_{12}\right)\right]$ and $\tilde{p}\left(r_{12}, r_{34}\right)=\max$ $\left[p\left(r_{12}, r_{34}\right), p\left(r_{34}, r_{12}\right)\right]$. Figures 8 and 9 show these symmetrized distributions at room temperature. Symmetrizing the distribution

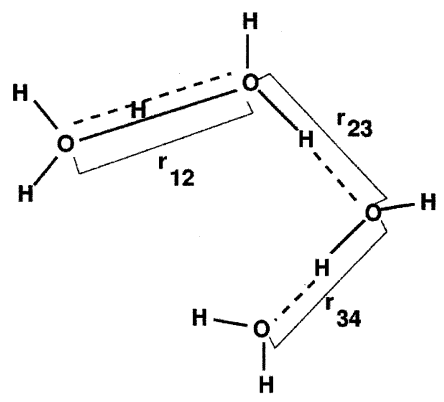

Figure 7. Distance definitions. $r_{12}$ is the oxygen-oxygen distance of the Zundel complex which contains the proton. $r_{23}$ and $r_{34}$ are nearest and second-nearest neighbors.

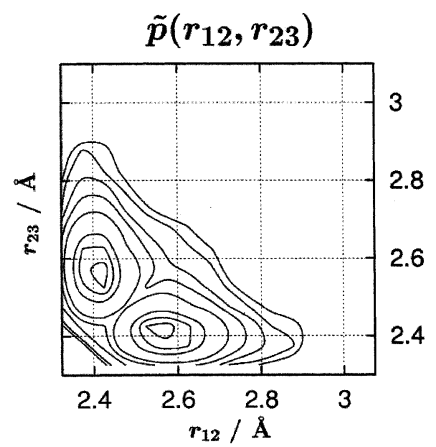

Figure 8. Probability distribution $\tilde{p}\left(r_{12}, r_{23}\right)$, which is the probability of finding two oxygen-oxygen distances. $r_{12}$ is the distance between the two oxygen atoms closest to the free proton, and $r_{23}$ is the oxygenoxygen distance between one of these oxygen atoms and a nearest neighbor.

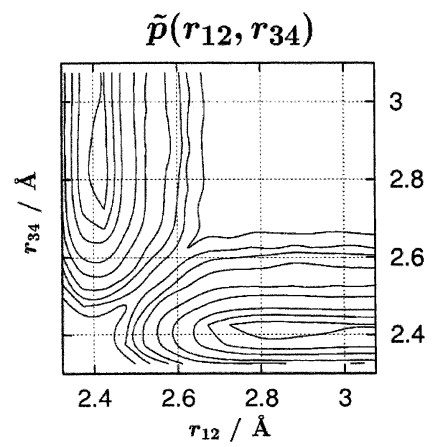

Figure 9. Probability distribution $\tilde{p}\left(r_{12}, r_{34}\right)$, which is the probability of finding two oxygen-oxygen distances. $r_{12}$ is the distance between the two oxygen atoms closest to the free proton, and $r_{34}$ is the oxygenoxygen distance between the first and second nearest neighbors.

in such a way is equivalent to taking, at every point, the minimum of the two free energy landscapes corresponding to the proton in the initial and final state. This procedure is motivated by the symmetry of the PT reaction. The saddle points of the distributions can be identified as transition states for the respective PT reactions in coordinate space, whereas the maxima correspond to the equilibrium states. With this interpretation, the free energy barrier for the PT reaction becomes: $\Delta A=$ $-k T \ln p$ (barrier) $/ p$ (maximum).

For PT to the nearest neighbor (Figure 8), we find $A=0.05$ $\mathrm{eV}$, and for PT to the second nearest neighbor (Figure 9), $\Delta A$ $=0.08 \mathrm{eV}$. This suggests, once more, that the rate-determining step is likely to be associated with the PT to a second nearest neighbor rather than to a nearest neighbor. 


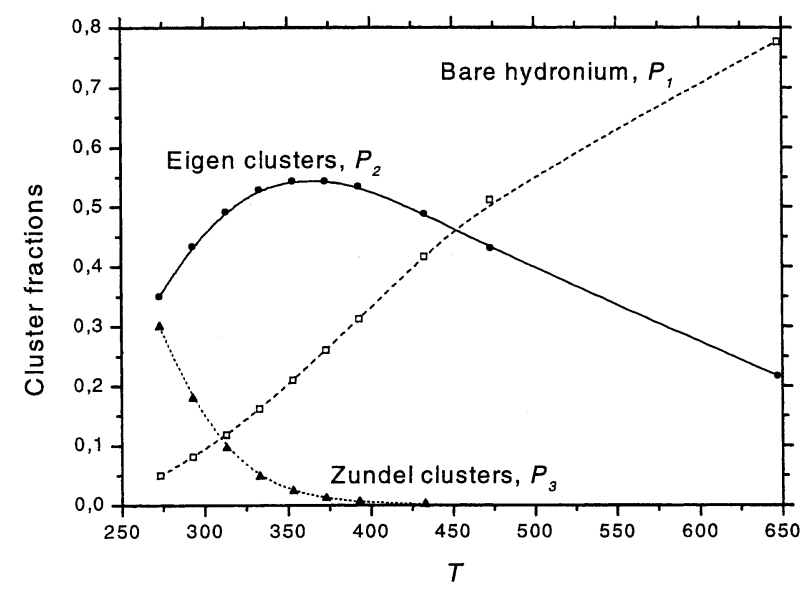

Figure 10. Cluster weights evaluated by Gierer and Wirtz. ${ }^{8}$

\section{Structural and Kinetic Properties, Integrated in Proton Mobility}

7.1. PT Cluster Weights Distributions $\boldsymbol{P}_{\boldsymbol{k}}$. As noted, aqueous proton mobility data over broad ranges of temperature recorded early by Eucken ${ }^{4}$ were analyzed by Gierer and Wirtz. ${ }^{8}$ They invoked a four-state model based on variable-size PT aqueous clusters in labile thermal equilibria. Three of the clusters correspond roughly to bare hydronium, the Eigen cluster, and the Zundel cluster and dominate proton conduction in most of the temperature range. The fourth cluster in their analysis is larger and contributes about $18 \%$ to the total at room temperature but disappears rapidly as the temperature rises. Inclusion of this component in our analysis would be straightforward but increases the number of a priori unknown parameters unwarrantedly.

Figure 10 shows the temperature variation of the three dominating cluster populations, $P_{1}, P_{2}$, and $P_{3},{ }^{8}$ and Figure 11 shows similar variations based on eq 7. The trend in Figure 11 accords broadly with the data for suitable parameters, i.e., small reaction enthalpies and entropies. Particularly, the Eigen and Zundel clusters dominate entirely around room temperature. As the temperature increases, the more composite Zundel clusters decay and are converted to the Eigen clusters, the population of which therefore first rises. This pattern is supported by the MD simulations (see Figure 4). On further temperature increase, the Eigen clusters also decay, and the bare hydronium ion gradually comes to dominate.

7.2. Mobility. The temperature-dependent cluster distribution, substantiated by the MD simulations, provides the mechanistic basis for the earlier estimates. ${ }^{8}$ The clusters and the MD calculations thus provide the structural and dynamic details needed for application of quantum mechanical PT theory toward a description of the proton mobility. A summary of the PT formalism for all the scenarios considered in section 6 and shown in Figure 5 is given in Appendices A-C. The formalism is given in a form which is straightforward to use, even analytically, at different levels of sophistication as warranted by the focus and detail needed. A complete description of the proton mobility by a combination of the MD-supported cluster populations and the PT formalism, however, still requires a considerable number of parameters. We show instead in a simpler way how this combination can both be brought to reproduce the observed temperature-dependent mobility and lead to insight and determination of some of the central parameters, which are difficult to access in other ways.

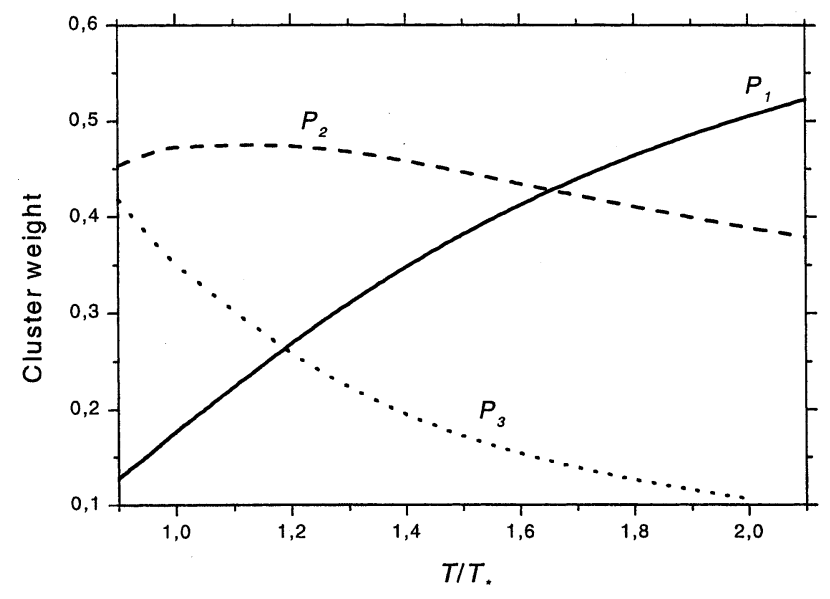

Figure 11. Typical cluster weights of a three-state model. Calculation via eq 7 with $A_{12}=0.22, A_{23}=0.1, E_{12}=2.5 k_{\mathrm{B}} T, E_{23}=2 k_{\mathrm{B}} T$ with $T=298.15 \mathrm{~K}$

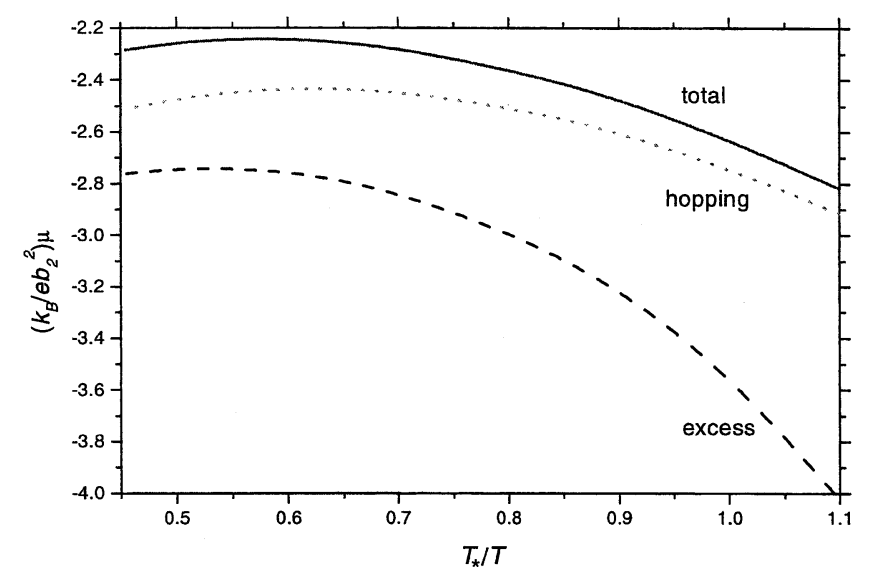

Figure 12. Typical theoretical plots of the measurable excess mobility, total mobility, and the contribution to mobility due to proton hopping between the charge-transfer clusters. Calculation via eqs $7-10 . b_{i}=$ $a_{i}{ }^{2} N_{i}\left(\omega_{i}\right) /(4 \pi) \kappa_{i},\left(i=1,2\right.$, and 3). Parameters: $A_{12}=0.22, A_{23}=0.1$, $E_{12}=2.5 k_{\mathrm{B}} T, E_{23}=2 k_{\mathrm{B}} T, w_{1}=1.25, w_{2}=2.5, w_{3}=4,\left(b_{1}\right) /\left(b_{2}\right)=$ $0.15,\left(b_{3}\right) /\left(b_{2}\right)=4, T_{*}=298.15 \mathrm{~K}$.

The use of comprehensive PT theory is first illuminated below in a general but still accurate form. In the next two subsections, we show how specific structural parameters of importance for the apparent activation energy and kinetic deuterium isotope effect can be obtained.

Figure 12 shows the total mobility, the measurable excess mobility, and the mobility caused only by proton hopping, calculated from eqs $7-10$ using a set of parameter values chosen to reveal similar trends as in the experimental data. It is noted first that the excess mobility passes through a maximum at high temperature. This follows the data in refs $6-8$, which were obtained at elevated pressures. Although numerically different, the proton conductances of the three PT channels are, second, still broadly similar. The excess proton mobility therefore follows closely the temperature variation of the populations of the three molecular entities, i.e., the bare hydronium ion, the Eigen cluster, and the Zundel cluster. As noted, the Zundel cluster population decays rapidly with increasing temperature. The decay is, however, via the Eigen clusters. The excess mobility thus first rises with increasing temperature and then begins to decline at still higher temperatures, when also the Eigen clusters decay into bare hydronium ions. The lower conductivity of the latter is partly compensated by the activated 
nature of all of the processes, but the maximum in the temperature variation shows that the variation of the population distribution clearly seems to dominate. Note, however, that the extremum in the total mobility is a result of a subtle interplay between a number of structural and kinetic factors. One cannot insist that it must be observed in reality.

7.3. Activation Gibbs Free Energies and Proton-Transfer Distances. As noted, there is no single activation (Gibbs free) energy, because the proton mobility is mediated by different clusters, see eq 3. PT theory can, however, rationalize the activation (free) energy of the different channels. We shall illustrate this for the Eigen and Zundel clusters

The complete activation free energy formalism is given in Appendices $\mathrm{A}-\mathrm{C}$. The activation free energies for the symmetric processes in question (zero driving force) are composed of two contributions. One is the total nuclear reorganization energy of the intramolecular solvation water clusters of the hydronium or Zundel ions including the bulk water solvent. The other one is the variation of the resonance splitting of the ground-state proton vibrational levels via motion along the intermolecular $\mathrm{O}-\mathrm{O}$ stretching mode in the potential $V_{\mathrm{O}-\mathrm{O}}(R)$, cf. eq 19. This effect can be important for the kinetic deuterium isotope effect, addressed below. We can disentangle these effects by the simple consideration below. In this way, we can estimate the activation Gibbs free energy, the contribution of each of the intramolecular terms, and the distance, $R_{\mathrm{s}}$, between the two neighboring oxygen atoms in the transition state in the Eigen or Zundel cluster.

The equation which determines $R_{\mathrm{s}}$ is, approximately

$$
\frac{\partial V_{\mathrm{O}-\mathrm{O}}}{\partial R}=\frac{1}{2} \frac{\partial \Delta E_{\mathrm{h}}}{\partial R}
$$

where $V_{\mathrm{O}-\mathrm{O}}$ is the interaction potential describing the hydrogen bond and $\Delta E_{\mathrm{h}}$ is the resonance splitting of the ground state proton energy levels in the potential which determines the proton motion between two oxygen atoms. This motion is the antisymmetric translation of the proton donor and acceptor moieties and is fully classical. $\Delta E_{\mathrm{h}}$ depends approximately exponentially on $R$

$$
\Delta E_{\mathrm{h}}=\Delta E_{\mathrm{h}}^{0} \exp \left[-\gamma\left(R-R_{\mathrm{HB}}\right)\right]
$$

where $R_{\mathrm{HB}}$ is the equilibrium hydrogen bonded $\mathrm{O}-\mathrm{O}$ distance in the initial (reactant) state. $\gamma$ (in $\AA^{-1}$ ) is a decay factor, which represents the proton localization at a given site. The Lennard Jones potential is, by and large, used for the bare interaction of two oxygen atoms

$$
V_{\mathrm{O}-\mathrm{O}}(R)=\frac{C_{12}}{R^{12}}-\frac{C_{6}}{R^{6}}
$$

$V_{\mathrm{O}-\mathrm{O}}(R)$ incorporates the effect of the proton between the two oxygen atoms on their mutual interaction. We shall still use the Lennard Jones potential form but take those values of the parameters $C_{12}$ and $C_{6}$ that reproduce the value of $R_{\mathrm{HB}}$, as obtained from the condition $\partial V_{\mathrm{O}-\mathrm{O}} / \partial R=0$. With $C_{6}=27.13$ $\mathrm{eV} \AA,{ }^{6}$ the value of the simulation water model, ${ }^{25}$ we obtain $C_{12}=13.56 R_{\mathrm{HB}}{ }^{6} \mathrm{eV} \AA^{12}$.

Appropriate values of $R_{\mathrm{HB}}$ range from $2.8 \AA$ for free water to about $2.4 \AA$ in the Zundel ion, whereas $\gamma$ ranges from 2 to $5 \AA^{-1}$. For $\Delta E_{\mathrm{h}}^{0} \approx 0.1 \mathrm{eV}$, this gives the values of $R_{\mathrm{s}}$ in Table 1. Substituting $R_{\mathrm{s}}$ into eq 28

$$
G^{\neq}=\frac{1}{2} D+V_{\mathrm{O}-\mathrm{O}}\left(R_{\mathrm{s}}\right)-V_{\mathrm{O}-\mathrm{O}}\left(R_{\mathrm{HB}}\right)-\frac{1}{2} \Delta E_{\mathrm{h}}\left(R_{\mathrm{s}}\right)
$$

TABLE 1: Donor-Acceptor O-O Distances at the Transition State, $\boldsymbol{R}_{\mathrm{S}}(\AA)$, at Different Equilibrium Distances, $\boldsymbol{R}_{\mathrm{HB}}(\AA)$, and Decay Factors $\gamma\left(\AA^{-1}\right)$, eq $\mathbf{2 6}^{a}$

\begin{tabular}{ccclll}
\hline$R_{\mathrm{HB}}=2.8 \AA$ & & & & & \\
$\gamma$ & 2 & 3 & 3.5 & 3.7 & 4 \\
$R_{\mathrm{s}}-R_{\mathrm{HB}}$ & 0.24 & 0.38 & 0.48 & 0.53 & 0.61 \\
$G^{\ddagger}$ & $\approx 0$ & 0.02 & 0.08 & 0.13 & 0.27 \\
$R_{\mathrm{HB}}=2.6 \AA$ & & & & & \\
$\quad \gamma$ & 2 & 3 & 4 & 4.2 & 4.5 \\
$\quad R_{\mathrm{s}}-R_{\mathrm{HB}}$ & 0.15 & 0.24 & 0.32 & 0.40 & 0.47 \\
$\quad G^{\ddagger}$ & $\approx 0$ & $\approx 0$ & 0.003 & 0.03 & 0.15 \\
$R_{\mathrm{HB}}=2.5 \AA$ & & & & & \\
$\gamma$ & 3 & 4 & 4.5 & 4.9 & 5.0 \\
$\quad R_{\mathrm{s}}-R_{\mathrm{HB}}(\AA)$ & 0.08 & 0.20 & 0.34 & 0.42 & 0.44 \\
$G^{\ddagger}$ & $\approx 0$ & $\approx 0$ & 0.03 & 0.12 & 0.16
\end{tabular}

${ }^{a} G^{\ddagger}(\mathrm{eV})$ is the activation free energy calculated from eq 28 by neglecting $D$ (see text).

(cf. eq 19) and neglecting $D\left(D=D_{\mathrm{HB}}, D_{\mathrm{Z}}\right)$, which is small, we obtain the activation free energy values collected in Table 1. In comparison, the experimental value of $G^{\neq}$is $0.11 \mathrm{eV} .6,8$ The PT parameters in Table 1 prompt the following observations:

(a) $\gamma=4.5-4.9 \AA^{-1}$ accords with the experimental activation (Gibbs free) energy. This $\gamma$ range is suitable for proton tunneling between shallow hydron double wells.

(b) The resulting PT distance ranges from $\approx 0.30 \AA$ when $R_{\mathrm{HB}}=2.8 \AA$ in "free" water to less than $0.1 \AA$ in the strongly hydrogen bonded Zundel complex. Significant thermal deformation along the $\mathrm{O}-\mathrm{O}$ stretching mode thus occurs prior to proton tunneling, which is subjected to much smaller displacements than corresponding to equilibrium displacement along the proton translational coordinate.

(c) The distances in Table 1 and the conclusion in (b) are based on eq 28 and thermal activation dominated by the $\mathrm{O}-\mathrm{O}$ gating mode. Significant activation in other nuclear modes would leave less need for gating mode stretching in order to reproduce the experimental activation free energy. The PT distance at the saddle point with respect to the local and environmental nuclear modes would therefore also be longer.

(d) The small tunneling distance accords with facile PT, gated by the $\mathrm{O}-\mathrm{O}$ stretching mode. This also leads to a small KIE, cf. below.

Altogether, the estimates in Table 1, based on eqs 25-28 point to the consistency of the microscopic PT picture presented above and accordance with the structural information about the Eigen and Zundel complexes.

7.4. Kinetic Deuterium Isotope Effect of Aqueous Proton Mobility. The kinetic deuterium and tritium isotope effect (KIE) in chemical proton, deuteron, and triton (overall hydron) tunneling has been recognized for decades as a crucial mechanistic approach to chemical hydron and hydrogen atom reaction dynamics. ${ }^{50,51,52} \mathrm{KIE}$ perspectives also emerge from the formalism and models in previous sections. There are only few data for excess deuteron mobility. These give a small value close to $\sqrt{ } 2,8,53-55$ close to the value for water molecular rotation. This suggests that different $\omega_{\text {eff }}$ in proton and deuteron transfer, eq 8 , constitutes a significant contribution, but we wish to address more closely the overall physical nature of the KIE in strongly interacting proton donor-acceptor systems such as the Eigen and Zundel complexes.

It is useful first to note briefly the KIE behavior in the weakcoupling diabatic limit (with respect to the proton-environmental separation). The origin of the KIE in PT is conspicuous and conceptually straightforward in this limit. The dominating KIE 
feature here is the isotope dependence of the transmission coefficient, $\kappa$, eq 8 , which directly reflects the hydron tunneling behavior. The following $\kappa_{\mathrm{P}}$ form applies $(\mathrm{P}=\mathrm{H}$ and $\mathrm{D})$, eq 26

$$
\kappa_{\mathrm{P}}=\kappa_{\mathrm{P}}^{0} \exp \left[-2 \gamma_{\mathrm{P}}\left(R_{\mathrm{s}}^{\mathrm{P}}-R_{\mathrm{HB}}\right)\right]
$$

As $\gamma_{\mathrm{H}}<\gamma_{\mathrm{D}}$, proton tunneling is more favorable than heavier deuteron tunneling i.e., $\kappa_{\mathrm{H}} \gg \kappa_{\mathrm{D}}$. As deuteron tunneling is significantly disfavored relative to proton tunneling, additional $\mathrm{O}-\mathrm{O}$ mode activation energy in deuteron tunneling is also energetically affordable as this decreases the tunneling distance. The diabatic hydron tunneling distance is therefore in general smaller for the heavier than for the lighter isotope.

However, we do not deal with the diabatic limit in the case of proton conductivity in water. MD and electronic structure computations (e.g., refs 28, 30, 31, 37, 38, and 25, 40-44) show instead that the interaction between the hydron donor and acceptor entities in the Eigen and Zundel clusters is strong. Hydron transfer is therefore well within the fully adiabatic limit of strong donor-acceptor interaction. In this limit, proton tunneling is not directly conspicuous, because by optimal fluctuations the environment prepares a minimal barrier (almost no barrier) for the proton. This has a cost in free energy which determines the activation free energy, whereas the under-barrier tunneling prefactors $\kappa_{\mathrm{H}}, \kappa_{\mathrm{D}} \rightarrow 1$. All in all, the KIE no longer originates from the transmission coefficient. The origin of the $\mathrm{KIE}$ in the adiabatic limit is more subtle and two other contributions instead determine the KIE.

One is the isotope dependence of $\omega_{\text {eff }}$, rooted in hindered translational and rotational mode frequencies. (This effect is unimportant in the diabatic limit). The translational modes involve motion of a solvent water molecule as a whole, with an isotope effect of $\omega_{\text {eff }}^{\mathrm{H}} / \omega_{\text {eff }}^{\mathrm{D}}$ of $\approx 18 / 16$. Thus, if the PT was provided only by the vehicle mechanism, i.e, motion of $\mathrm{H}_{3} \mathrm{O}^{+}$ as a whole, KIE would have been $\approx 1.1$. The Grotthus elements of PT are rather determined by the rotational modes. The latter rest largely on hydron motion around a stationary oxygen atom with a frequency $\omega_{\text {eff }}$ following roughly the square root of the hydron mass dependence, i.e., $\omega_{\text {eff }}^{\mathrm{H}} / \omega_{\text {eff }}^{\mathrm{D}} \approx \sqrt{ } 2$. This is close to the experimental value. Hindered molecular water rotation therefore constitutes a competitive contribution to the KIE of adiabatic PT in the Eigen and Zundel clusters (in accordance with conclusions derived by Hynes from the analysis of PT in small water clusters around an Eigen ion ${ }^{26,27}$ ).

The second important factor contributing to the KIE is the isotope dependent resonance splitting of the reduced potential surfaces spanned by the intramolecular and solvent coordinates, $\Delta E_{\mathrm{h}}\left(x_{\mathrm{s}}, y_{\mathrm{s}}, R_{\mathrm{s}}\right)$, eqs 16,17 , and 19 . This contribution is only important in the adiabatic limit and generally a competitive contribution to the KIE. Resonance splitting is caused by hydron tunneling in the double-well potential spanned by the proton coordinate $q$ (Figure 13). Less favorable deuteron transfer implies that the ground and first excited deuteron vibrational level splitting is smaller than for the proton levels. The reduced potential surface splitting is thus smaller for deuteron than for proton transfer. Hence, the activation free energy as determined by the intramolecular and solvent nuclear modes is larger for deuteron than for proton transfer, however, with a negligible effect on the under-barrier tunneling factor, because the proton barrier is very low.

This view is illustrated in Figure 13. The top figure shows the hydron stretching mode splitting for the two isotopes along the hydron coordinate, at the transition state with respect to the
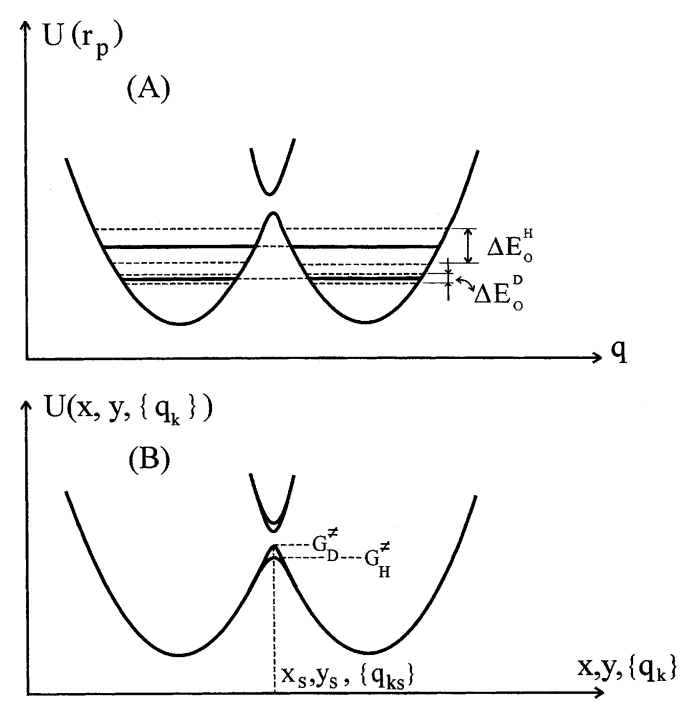

Figure 13. Kinetic deuterium isotope effect (schematic). (A) Splitting of the ground-state proton and deuteron vibrational energies in the hydrogen potential at fixed heavy nuclear coordinates. The deuteron level is the lower because of the lower vibrational energy. The deuteron splitting is also the smallest because of the heavier tunneling mass. (B) Lowering of the activation Gibbs free energy along the heavy nuclear coordinates by the hydron splitting. The activation energy lowering is larger for proton than for deuteron transfer because of the larger tunnel splitting and more facile tunneling.

heavy nuclear coordinates, $x_{\mathrm{s}}, y_{\mathrm{s}}, R_{\mathrm{s}}$, cf. eqs 16,17 , and 19 . The bottom figure shows how this carries over to the isotope dependence of the activation (free) energy along the intramolecular and solvent coordinates. The resonance splitting contribution to the KIE is then rooted in hydron tunneling as in the diabatic limit, but tunneling appears quite differently, namely, in the activation free energy and not in the preexponential tunneling factor as in the diabatic limit. The view of quantum mechanical PT in the adiabatic limit has an apparent resemblance to classical views of chemical KIE's as the loss of zero-point vibrational energy when the hydron is displaced from equilibrium to the transition state..$^{50-52}$ The KIE is reflected in isotope dependence of the activation (free) energy in both cases, but the origin of the activation Gibbs free energy difference is different. In the classical view, the isotope dependence is entirely determined by proton and deuteron motion, whereas the environmental nuclear motion is largely disregarded. In the adiabatic limit of quantum mechanical PT, the activation (free) energies of proton and deuteron transfer are viewed as determined by the environmental nuclear motion modified by isotope dependent resonance splitting, caused by quantum mechanical hydron tunneling behavior.

Eucken $^{4}$ reported small, temperature-dependent deuterium isotope effects in the cluster populations, $P_{1}, P_{2}$, and $P_{3}$, eq 3-5. The isotope effects in $P_{1}$ and $P_{2}$ are larger than unity but smaller than unity for $P_{3}$, i.e., $P_{k}^{\mathrm{H}} / P_{k}^{\mathrm{D}}>1$ for $k=1,2$ and $P_{3}^{\mathrm{H}} /$ $P_{3}^{\mathrm{D}}<1$. This could reflect a more stable hydrogen bond network for the heavier isotope. These isotope effects are smaller than $\sqrt{ } 2$ and $\sqrt{ } 2^{-1}$ and will be disregarded in the following.

The KIE of adiabatic symmetric PT processes such as in the Eigen and Zundel clusters takes the form, eq 8

$$
\mathrm{KIE} \equiv \frac{W_{\mathrm{H}}}{W_{\mathrm{D}}}=\frac{\omega_{\text {eff }}^{\mathrm{H}}}{\omega_{\text {eff }}^{\mathrm{D}}} \exp \left(\frac{G_{\mathrm{D}}^{\neq}-G_{\mathrm{H}}^{\neq}}{k_{\mathrm{B}} T}\right)
$$


where " $\mathrm{H}$ " refers to proton and " $\mathrm{D}$ " to deuteron transfer. The explicit isotope dependence of the activation Gibbs free energy difference in eq 30 is (eq 26)

$$
\begin{aligned}
& G_{\mathrm{D}}^{\neq}-G_{\mathrm{H}}^{\neq} \approx \Delta E_{\mathrm{h}}^{0 \mathrm{H}} \exp \left[-\gamma_{\mathrm{H}}\left(R_{\mathrm{s}}^{\mathrm{H}}-R_{\mathrm{HB}}^{\mathrm{H}}\right)\right]- \\
& \Delta E_{\mathrm{h}}^{0 \mathrm{D}} \exp \left[-\gamma_{\mathrm{D}}\left(R_{\mathrm{s}}^{\mathrm{D}}-R_{\mathrm{HB}}^{\mathrm{D}}\right)\right]
\end{aligned}
$$

where $R_{\mathrm{S}}$ is the value of $R$ in the transition state with respect to the $O-O$ stretching motion, cf. above. $R_{\mathrm{HB}}^{\mathrm{H}}$ and $R_{\mathrm{HB}}^{\mathrm{D}}$ are the equilibrium hydrogen bonded $\mathrm{O}-\mathrm{O}$ distances in the proton and deuteron bonded complex. $\Delta E_{\mathrm{h}}^{0 \mathrm{P}}, \mathrm{P}=\mathrm{H}$ and $\mathrm{D}$, refers to the resonance splitting at the saddle point with respect to the intramolecular and environmental coordinates, $x_{\mathrm{s}}$ and $y_{\mathrm{s}}$, at the equilibrium value of the $\mathrm{O}-\mathrm{O}$ stretching coordinate $R$ (eqs 18 and 20). Equations 30 and 31 reflect again the environmentally "gated" nature of the PT process. The hydron transfer distance is far too long, i.e., $0.5-0.6 \AA$, when tunneling is over the equilibrium displacement, but fluctuational motion along the $\mathrm{O}-\mathrm{O}$ stretching mode precedes, or "gates", $\mathrm{PT}$ over a much shorter and more favorable distance. This is at the expense of additional activation (free) energy from the $\mathrm{O}-\mathrm{O}$ mode deformation.

The isotope dependence of the activation Gibbs free energy, eqs 30 and 31 , is determined by the mass dependence of $\Delta E_{\mathrm{h}}^{\mathrm{P}}$ and $\gamma_{\mathrm{P}}$

$$
\begin{aligned}
G_{\mathrm{D}}^{\neq}-G_{\mathrm{H}}^{\neq} \approx & \Delta E_{\mathrm{h}}^{0 \mathrm{H}} \exp \left[-\gamma_{\mathrm{H}}\left(R_{\mathrm{s}}-R_{\mathrm{HB}}\right)\right] \times \\
& \left\{1-\frac{\Delta E_{\mathrm{h}}^{0 \mathrm{D}}}{\Delta E_{\mathrm{h}}^{0 \mathrm{H}}} \exp \left[-\left(\gamma_{\mathrm{D}}-\gamma_{\mathrm{H}}\right)\left(R_{\mathrm{s}}-R_{\mathrm{HB}}\right)\right]\right\}
\end{aligned}
$$

where the superscripts of $R_{\mathrm{S}}$ and $R_{\mathrm{HB}}$ have been omitted. $\Delta E_{\mathrm{h}}^{\mathrm{P}}, \Delta E_{\mathrm{h}}^{\mathrm{OP}}$ (eq 20), and $\gamma_{\mathrm{P}}$ are available for specific hydron potentials. ${ }^{35,56}$ Disregarding, further, the isotope dependence of $\Delta E_{\mathrm{h}}^{0 \mathrm{P}}$, eq 32 then reduces to

$$
\begin{aligned}
G_{\mathrm{D}}^{\neq}-G_{\mathrm{H}}^{\neq} \approx \Delta E_{\mathrm{h}}^{0} \exp \left[-\gamma_{\mathrm{H}}\left(R_{\mathrm{s}}-R_{\mathrm{HB}}\right)\right] \times \\
\\
\left\{1-\exp \left[-\left(\gamma_{\mathrm{D}}-\gamma_{\mathrm{H}}\right)\left(R_{\mathrm{s}}-R_{\mathrm{HB}}\right)\right]\right\}
\end{aligned}
$$

Equations 32 and 33 hold an intriguing implication, namely, that the adiabatic KIE decreases with increasing hydron transfer distance. (This is opposite to diabatic KIE, which is dominated by the preexponential hydron tunneling factor.) This "inverse" distance dependence of the KIE is caused by the decreasing resonance splitting of the potential surfaces with increasing transfer distance. The isotope dependence of the splitting therefore also decreases with increasing distance. This is the determining factor in the adiabatic limit (but quite unimportant in the diabatic limit). The overall distance dependence of the $\mathrm{KIE}$ is therefore nonmonotonic. At long distances, where the diabatic limit of weak interaction would have prevailed, the KIE decreases with decreasing distance, as the hydron donor and acceptor fragments are brought closer to each other. When the distance is small enough (as is commonly assumed and resulting from MD simulations for PT mobility in water), and the adiabatic limit of strong interaction takes over, the KIE behavior changes, and the KIE now increases with decreasing distance. A minimum in the distance dependence of the KIE is thus expected in the transition region between the diabatic and adiabatic limits. This minimum is not reached in PT acts of proton mobility, which accord with the adiabatic limit. The KIEis therefore expected to increase with decreasing transfer distance up to the intermolecular donor-acceptor distance where the hydron barrier vanishes altogether.

Equations 30-33 offer both an additional clue to the small $\mathrm{KIE}(\approx 1.4)$ in aqueous proton conductivity and kinematic insight into the PT mechanism. As aqueous nonclassical proton conductivity in the Eigen and Zundel complexes follows the strongly adiabatic limit, proton tunneling can only be reflected in the isotope dependent resonance splitting and activation (free) energy and not in the preexponential tunneling factor. The values of $R_{\mathrm{S}}-R_{\mathrm{HB}}$ from the analysis summarized in Table 1 leave PT distances of about $0.1 \AA$, based on equilibrium values of $R_{\mathrm{HB}}$ $\approx 2.5-2.6 \AA$, which accord with the Eigen and Zundel ion structures. Insignificant KIEs then emerge from eqs 32 and 33. Larger PT distances, say $0.2 \AA$, emerge when nuclear modes other than the $\mathrm{O}-\mathrm{O}$ gating mode contribute to the activation free energy. The resulting KIE is, however, smaller than 1.51.6 , i.e., close to the observed values for the proton conductivity and the water molecular librational frequency.

All in all, the KIE therefore also testifies that aqueous proton mobility is dominated by plain inertial mass effects, perhaps supplemented by subtle hydron tunneling features. The former appears in the mass dependence of the classical hydron transfer "attempt frequency", and the latter in hydron tunnelingcontrolled resonance splitting and activation Gibbs free energy. The KIE analysis also supports that aqueous proton conduction is dominated by reorganization in the $\mathrm{O}-\mathrm{O}$ gating mode and other local solvent modes. Reorganization and gating thus precedes the hydron transfer event along the proton translational coordinate, which itself follows in an almost barrierless fashion after the heavy nuclear mode preorganization and gating.

\section{Concluding Remarks}

Microscopic views of proton conduction in bulk water and confined heterogeneous environments, such as polymer electrolytes, should ultimately be resolved by MD simulations. As the molecular actors in these scenarios are engaged in the exchange of protons as quantum particles, the simulations should be at a level which includes the quantum mechanical nature of the proton, such as in Carr-Parinello simulations for bulk proton conductivity. We have shown that the most important elements of the proton dynamics can also be rationalized systematically and in a transparent fashion on the basis of contemporary concepts and formalism of the theory of charge-transfer processes in condensed media, supported by MD simulations of the environmental nuclear configurational dynamics. Charge transfer theory rests on the notion of transfer of charged particles such as electrons and protons between well-defined molecular donor and acceptor entities. Proton conduction in bulk aqueous solution and in confined membrane environments is carried out by rapidly interconverting proton transferring clusters subjected to fast mutual equilibrium fluctuations. PT within and between the clusters involves strong donor-acceptor interactions and shallow PT barriers in preorganized proton double-wells. These are significantly distorted from the proton double-well configurations at the equilibrium environmental nuclear configurations.

Properties of the proton transferring clusters were identified phenomenologically in impressive detail in classical works, when neither modern charge transfer theory nor powerful computational tools such as MD simulations existed. Composition and properties of proton transferring clusters have been largely supported by modern MD approaches at different levels. Thus, the notion of proton exchanging molecular entities, which interconvert rapidly, remains of considerable value and warrants the use of modern condensed-matter PT theory. With such 
views, we have shown that the powerful frames of PT theory carry over to and help to rationalize bulk aqueous proton mobility. The PT processes in the Eigen and Zundel clusters have been identified and found to accord with the adiabatic limit of strongly interacting donor and acceptor clusters. MD simulations have, further, identified the most important local nuclear reactive modes that have been the basis for the construction of potential free energy surfaces and the calculation of the activation free energy. The experimental value of the latter $(0.11 \mathrm{eV})$ can be reproduced by a class of models dominated by deformation of the intermolecular $\mathrm{O}-\mathrm{O}$ gating mode of the proton donor and acceptor. This offers a view of the fundamental $\mathrm{PT}$ process in clusters where major preorganization in the $\mathrm{O}-\mathrm{O}$ gating mode leads the proton to pass a shallow or negligible proton mode barrier. These views also open a rationale for the kinetic deuterium isotope effect. The KIE appears to be dominated by the preexponential librational frequency, $\omega_{\text {eff }}$, but may hold a small contribution from the isotope-dependent lowering of the activation free energy in the crossing region of the reduced potential free energy surfaces spanned solely by the heavy nuclear coordinates.

Disclosure of the nature of the PT elementary act in bulk proton conducting clusters, and development of bulk proton conductivity models hold, finally, perspectives for addressing the changes in the proton conductivity patterns when the water solvent is embedded in heterogeneous polymer electrolyte membranes or biological trans-membrane proton conducting channels. The pores in Nafion polymer electrolyte membranes are, for instance, formed by water uptake with subsequent dissociation of the $-\mathrm{SO}_{3} \mathrm{H}$ groups immobilized on the polymer side-chains and hydration of the resulting $-\mathrm{SO}_{3}{ }^{-}$groups. Water confined in such environments possesses orientational dynamics different from the bulk. The dynamics is impeded close to the pore surface in wide pores and almost frozen in narrow pores. This affects immediately the proton conducting performance of pores of different size and internal structure. Information about the pore hydration structure in proton conducting protein channels would undoubtedly assist to rationalize different aspects of the proton conducting properties, as the structure of such channels is mostly better known than the Nafion membrane structures. In all these exciting areas of proton conductivity, the models of water and solvated proton and their further development remain at the heart of the matter. Bulk water and solvated proton models must not, so to speak, be "undrinkable" before let into the relevant complex membrane or protein environments.

Acknowledgment. The authors are thankful to Margarita Rodnikova for useful discussions. Special thanks are due to Sean Walbran, whose code with minor modifications was used in these MD simulations. This work was supported by the grants of BMWi (Project No. 0327086), INTAS (Project No. 99-1093), and the Danish Technical Science Research Council (Contract No. 9801629). A.A.K. acknowledges the Royal Society of London for a Wolfson Research Merit Award. A.M.K. acknowledges the Alexander von Humboldt Foundation for a Humboldt Research Award and Wolfgang Schmickler at the University of Ulm for hospitality. E.S. and A.A.K. acknowledge computer time by John von Neumann Institute of Computing at Research Center Jülich.

\section{Appendix A}

Equations for the Saddle Point for the Hopping Mechanism I. The activation barrier is determined by the saddle point of the lower adiabatic free energy surface. The system of equations for the saddle point is ${ }^{34,35}$

$$
\begin{gathered}
(1-\alpha) \frac{\partial U_{\mathrm{i}}^{\mathrm{r}}}{\partial x}+\alpha \frac{\partial U_{\mathrm{f}}^{\mathrm{r}}}{\partial x}=\frac{\partial \Delta E_{h}}{\partial x}[\alpha(1-\alpha)]^{1 / 2} \\
(1-\alpha) \frac{\partial U_{\mathrm{i}}^{\mathrm{r}}}{\partial y}+\alpha \frac{\partial U_{\mathrm{f}}^{\mathrm{r}}}{\partial y}=\frac{\partial \Delta E_{\mathrm{h}}}{\partial y}[\alpha(1-\alpha)]^{1 / 2} \\
(1-\alpha) \frac{\partial U_{\mathrm{i}}^{\mathrm{r}}}{\partial R}+\alpha \frac{\partial U_{\mathrm{f}}^{\mathrm{r}}}{\partial R}=\frac{\partial \Delta E_{\mathrm{h}}}{\partial R}[\alpha(1-\alpha)]^{1 / 2} \\
U_{\mathrm{i}}^{\mathrm{r}}-U_{\mathrm{f}}^{\mathrm{r}}=\frac{(2 \alpha-1) \Delta E_{\mathrm{h}}}{2[\alpha(1-\alpha)]^{1 / 2}} \\
\alpha=\frac{\frac{\partial U_{\mathrm{i}}^{\mathrm{r}}}{\partial q_{k}}}{\frac{\partial U_{\mathrm{i}}^{\mathrm{r}}}{\partial q_{k}}-\frac{\partial U_{\mathrm{f}}^{\mathrm{r}}}{\partial q_{k}}}
\end{gathered}
$$

The value of $\alpha$ satisfying these equations is the symmetry factor, which characterizes the symmetry of the transition configuration and the dependence of activation (free) energy on the driving force. For the transition between identical states, hereafter called symmetric transitions, the driving force is zero. In the transition configuration, $U_{\mathrm{i}}^{\mathrm{r}}=U_{\mathrm{f}}^{\mathrm{r}}$, and eq A4 gives $\alpha=0.5$. In the absence of conspicuous external electric field effects, the elementary transitions of the proton in the Eigen and Zundel clusters are between symmetric states, and only the case $\alpha=$ 0.5 is of interest.

\section{Appendix B}

Model Potentials for the Hopping Mechanism I. The molecular potentials must be known for the calculation of the activation barrier. Morse and exponential functions are often used as model potentials: $:^{57-60}$

$$
\begin{gathered}
u_{1}=D_{x}\left[1-\mathrm{e}^{-\gamma_{x}\left(x-x_{0}\right)}\right]^{2} \\
u_{2}=D_{y}\left[1-\mathrm{e}^{-\gamma_{\mathrm{y}}\left(y-y_{0}\right)}\right]^{2} \\
V_{\mathrm{w} 1-\mathrm{O}}^{\mathrm{i}}(x)=\zeta_{x} D_{x} \mathrm{e}^{-2 \gamma_{x} \rho_{x}\left(x-x_{0}\right)} \\
V_{\mathrm{w} 2-\mathrm{O}}^{\mathrm{f}}(y)=\zeta_{y} D_{y} \mathrm{e}^{-2 \gamma_{y} \rho_{y}\left(y-y_{0}\right)}
\end{gathered}
$$

In general, the parameters of all of the potentials are different. However, because the transition is symmetric

$$
\begin{gathered}
D_{x}=D_{y}=D_{\mathrm{HB}} ; \quad \zeta_{x}=\zeta_{y}=\zeta \\
\gamma_{x}=\gamma_{y}=\gamma ; \quad \rho_{x}=\rho_{y}=\rho
\end{gathered}
$$

Hereafter, for simplicity, we set the dimensionless coefficients $\zeta=\rho=1$. The equations for the saddle point for the $x$ and $y$ modes then yield $X=Y=1 / 2$, where

$$
X=\mathrm{e}^{-\gamma\left(x-x_{0}\right)} ; \quad Y=\mathrm{e}^{-\gamma\left(y-y_{0}\right)}
$$

$R_{\mathrm{S}}$ is determined from the equation

$$
\frac{\partial V_{\mathrm{O}-\mathrm{O}}}{\partial R} \approx \frac{1}{2} \frac{\partial \Delta E_{\mathrm{I}}}{\partial R}
$$

The use of these quantities in eq 17 leads to eq 19. 


\section{Appendix C}

Equations for the Saddle Point for the Mechanism II. Using eqs $18-20$ for the free energy surfaces and eqs $\mathrm{A} 1-\mathrm{A} 5$, we write the equations for the saddle point for $R_{12}$ and $R_{23}$ as

$$
\begin{gathered}
\frac{\partial u_{12}}{\partial R_{12}}+\frac{\partial V_{12}^{\mathrm{f}}}{\partial R_{12}}+\frac{\partial u_{23}}{\partial R_{12}}=\frac{\partial \Delta E_{\mathrm{Z}}}{\partial R_{12}} \\
\frac{\partial u_{12}}{\partial R_{23}}+\frac{\partial V_{23}^{\mathrm{i}}}{\partial R_{23}}+\frac{\partial u_{23}}{\partial R_{23}}=\frac{\partial \Delta E_{\mathrm{Z}}}{\partial R_{23}}
\end{gathered}
$$

The equation for the saddle point values of $q_{k}$ is the same as eq A.5 with $\alpha=0.5$.

If we use Morse/exponential functions for the molecular potentials similar to those in Appendix B with $D=D_{\mathrm{Z}}$ and exponential dependence of the resonance energy splitting on the coordinates of the reactive modes, then

$$
\Delta E_{\mathrm{Z}} \approx \Delta E_{0} \exp \left[-\gamma\left(R_{12}-R_{12}^{0 \mathrm{i}}\right)\right] \exp \left[-\gamma\left(R_{23}-R_{23}^{0 \mathrm{f}}\right)\right]
$$

The solution of the equations for the saddle point is

$$
X=Y=\frac{D_{\mathrm{Z}}}{2 D_{\mathrm{Z}}-\Delta E_{0}}
$$

where

$$
X=\mathrm{e}^{-\gamma\left(R_{12}-R_{12}^{0 \mathrm{i}}\right)} ; \quad Y=\mathrm{e}^{-\gamma\left(R_{23}-R_{23}^{0 \mathrm{f}}\right)}
$$

Equations for the Saddle Point and Activation Gibbs Free Energy for PT from a Given Zundel Ion to a Different Zundel Ion. From the potentials

$$
\begin{gathered}
U_{\mathrm{i}} \approx u_{12}^{\mathrm{i}}\left(r_{12}\right)+V_{23}^{\mathrm{i}}\left(r_{23}\right)+V_{34}^{\mathrm{i}}\left(r_{34}\right) \\
U_{\mathrm{f}} \approx V_{12}^{\mathrm{f}}\left(r_{12}\right)+V_{23}^{\mathrm{f}}\left(r_{23}\right)+u_{34}^{\mathrm{f}}\left(r_{34}\right) \\
V_{23}^{\mathrm{i}}\left(r_{23}\right) \equiv V_{23}^{\mathrm{f}}\left(r_{23}\right) \\
u_{12}=u_{34}=D\left(1-\mathrm{e}^{-\gamma\left(r-r_{0}\right)}\right)^{2} \\
V_{12}=V_{34}=D \mathrm{e}^{-2 \gamma\left(r-r_{0}\right)} \\
\Delta E=\Delta E_{0} \mathrm{e}^{-\gamma\left(r_{12}-r_{12}^{0}\right)} \mathrm{e}^{-\gamma\left(r_{34}-r_{34}^{0}\right)} \mathrm{e}^{-\gamma\left(r_{23}-r_{23}^{0}\right)} \\
V_{23}=D\left(1-\mathrm{e}^{-\gamma\left(r_{23}-r_{23}^{0}\right)}\right)^{2}
\end{gathered}
$$

we obtain

$$
\begin{gathered}
\frac{\partial u_{12}^{\mathrm{i}}}{\partial r_{12}}+\frac{\partial V_{12}^{\mathrm{f}}}{\partial r_{12}}=\frac{\partial \Delta E}{\partial r_{12}} \\
2 \frac{\partial V_{23}^{\mathrm{i}}}{\partial r_{23}}=\frac{\partial \Delta E}{\partial r_{23}} \\
\frac{\partial u_{34}^{\mathrm{f}}}{\partial r_{34}}+\frac{\partial V_{34}^{\mathrm{i}}}{\partial r_{34}}=\frac{\partial \Delta E}{\partial r_{34}} \\
D \frac{\partial}{\partial X}(1-X)^{2}-2 D X^{2}=-2 \Delta E_{0} X Y R \\
D(1-X) 2 X-2 D X^{2}=-2 \Delta E_{0} X Y R \\
D(1-Y) 2 Y-2 D Y^{2}=-2 \Delta E_{0} X Y R \\
4 D_{23}(1-R) R=-2 \Delta E_{0} X Y R
\end{gathered}
$$

$$
\begin{gathered}
D X(1-2 X)=-\Delta E_{0} X Y R \\
D Y(1-2 Y)=-\Delta E_{0} X Y R \\
2 D_{23} R(1-R)=-\Delta E_{0} X Y R
\end{gathered}
$$

with

$$
\begin{aligned}
X=\mathrm{e}^{-\gamma\left(r_{12}-r_{012}\right)} ; \quad Y=\mathrm{e}^{-\gamma\left(r_{34}-r_{034}\right)} & (1-2 X)=-\Delta Y R \\
& (1-2 Y)=-\Delta X R \\
& (1-R)=-\delta Y X Y \\
\Delta= & \Delta E_{0} / D ; \delta=\Delta E_{0} / 2 D_{23}
\end{aligned}
$$

For

$$
\begin{gathered}
\mathrm{X}=\mathrm{Y} \\
(1-2 X)=-\Delta X R \\
(1-R)=-\delta X^{2}
\end{gathered}
$$

the equation for $\mathrm{X}$ is

$$
\Delta \delta X^{3}+(\Delta-2) X+1=0
$$

A solution exists if

$$
2-\Delta>3 \Delta \delta
$$

At $\Delta, \delta \ll 1$ the approximate solution has the form

$$
\begin{gathered}
X \approx \frac{1}{2}+x \\
x=\frac{\Delta}{4} \\
R \approx 1+\frac{\delta}{4}
\end{gathered}
$$

and the activation barrier is

$$
G^{\neq} \approx \frac{1}{E_{\mathrm{r}}}+\frac{D_{\mathrm{Z}}}{2}+\frac{D_{\mathrm{Z}} \Delta^{2}}{8}+D_{23} \frac{\delta^{2}}{16}-\frac{\Delta E}{2}
$$

\section{Appendix D}

List of the Key Symbols

$a_{\mathrm{i}}=\mathrm{PT}$ transfer distance

$A_{i j}=$ entropy factors

$D=$ self-diffusion coefficient

$e=$ charge of proton

$E_{i j}=$ activation enthalpies

$E_{\mathrm{p}}=$ proton energy

$E_{\mathrm{HB}}=$ dissociation energy of hydrogen bond

$D_{\mathrm{Z}}=$ dissociation energy of the Zundel complex

$\Delta E_{0}=$ resonance splitting at equilibrium of reactive coordinates

$\Delta E_{\mathrm{h}}$ and $\Delta E_{\mathrm{Z}}=$ quantum mechanical splitting of proton energy levels for the mechanisms I and II, respectively

$G^{\neq}=$activation Gibbs free energy

$\gamma=$ decay factor

$h=$ Planck's constant, $\hbar=h / 2 \pi$

$\kappa=$ transmission coefficient for transferring proton

$k_{\mathrm{B}}=$ Boltzmann's constant

$k_{i j}=$ rate constants

$K_{i j}=$ equilibrium constants 
$\mu=$ mobility

$P_{i}=$ relative concentration of clusters

$\left\{q_{\mathrm{k}}\right\}=$ reactive outer-sphere coordinates

$q=$ proton coordinate

$R_{12}=\mathrm{O}-\mathrm{O}$ distance between the two oxygen atoms within the Zundel complex

$R_{13}=\mathrm{O}-\mathrm{O}$ distance between any one oxygen atom of the Zundel complex and a neareast neighbor

$R_{14}=\mathrm{O}-\mathrm{O}$ distance between a nearest and second nearest neighbor of a Zundel complex

$R_{\mathrm{HB}}=$ hydrogen bond distance

$R_{\mathrm{S}}=\mathrm{O}-\mathrm{O}$ distance at the transition state

$t=$ time

$T=$ temperature

$W_{\mathrm{i}}^{\mathrm{s}}=$ free energy of outer sphere water polarization

$U_{\mathrm{i}}^{\mathrm{r}}=$ reduced free energy

$\omega_{\text {eff }}=$ effective vibrational frequency

$W_{\mathrm{i}}=$ transition probability per unit time

$\left\langle x^{2}(t)\right\rangle=$ mean square displacement

$\alpha=$ symmetry factor

\section{References and Notes}

(1) Erdey-Gruz, T. Transport Phenomena in Electrolyte Solutions; Adam Higler: London, 1974.

(2) Johnston, J. J. Am. Chem. Soc. 1909, 31, 987.

(3) Noyes, A. J. Chim. Phys. 1910, 6, 505; Z. Phys. Chem. Phys. 1910, 70,356 .

(4) Eucken, A. Z. Elektrochem. 1948, 52, 6; 1948, 52, 255.

(5) Gierer, A.; Wirtz, K. J. Chem. Phys. 1949, 17, 745.

(6) Gierer, A. Z. Naturforsch. 1950, $5 a, 581$.

(7) Franck, E. U.; Hartmann, D.; Hensel, F. Discuss. Faraday Soc. $1965,39,200$

(8) Gierer, A.; Wirtz, K. Ann. Phys. 1949, 6, 17.

(9) Kohlrausch, F.; Heydweiller, A. Ann. Phys. Chem. (Neue Folge) 1894, 53, 14.

(10) Uosaki, K.; Okazakai, K.; Kita, H. J. Electroanal. Chem. 1990, 287, 163.

(11) Cappadonia, M.; Erning, J. W.; Stimming, U J. Electroanal. Chem. 1994, 376, 189

(12) Cappadonia, M.; Erning, J. W.; Niaki, S. M. S.; Stimming, U. Solid State Ionics 1995, 77, 65.

(13) Gottesfeld, S.; Zawodzinski, T. A. Polymer Electrolyte Fuel Cells. In Advances in Electrochemical Science and Engineering; Alkire, R. C., Gerischer, H., Kolb, D. M., Tobias, C. W., Eds.; Wiley-VCH: Weinheim, Germany, 1997; Vol. 5, pp 195-301.

(14) Such a conjecture is justified if the proton distribution is delocalized within the pore. The precise proton distribution is not known. Mean field theory [Eikerling, M.; Kornyshev, A. A.; Kuznetsov, A. M.; Ulstrup, J.; Walbran, S. J. Phys. Chem. B 2001, 105, 3646] suggests a smooth radial distribution with a finite proton population in the centre of the pore, where the protons would move almost as in the bulk. Simulation results [Spohr, E.; Commer, P.; Kornyshev, A. A. J. Phys. Chem. B 2002, 106, 10560] are less unambiguous and show strong pinning of protons to pointlike immobile counter charges on the walls of the pore. Proton localization is much weaker for distributed and fluctuating negative charges in the models of real side chains.

(15) von Grotthuss, C. J. D. Ann. Chim. 1806, LVIII, 54.

(16) Bernal, J. D.; Fowler, R. H. J. Chem. Phys. 1933, 1, 515.

(17) Eigen, M.; de Maeyer, L. Proc. R. Soc. (London) 1958, A247, 505.

(18) Conway, B. E.; Bockris, J. O'M; Linton, H. J. Chem. Phys. 1956, 31,834 .
(19) Zundel, G.; Metzger, H. Z. Phys. Chem. 1968, 58, 225.

(20) Agmon, N. Chem. Phys. Lett. 1995, 244, 456.

(21) For overviews, see articles in: Chem. Rev. 1996, 96, 2237-3042. Special Issue on Bioinorganic Enzymology (Holm, R. H., Solomon, E. I., Eds.).

(22) For an overview, see: Kuznetsov, A. M.; Ulstrup, J. Can. J. Chem. 1999, 77, 1085.

(23) Proton Conducting Membrane Fuel Cells; Gottesfeld, S., Fuller, T. F., Eds.; The Electrochemistry Society Inc.: Pennington, NJ, 1999; Proc. Vol. 98-27.

(24) Proton Conductors; Colomban, P., Ed.; Cambridge University Press: Cambridge, 1992.

(25) Walbran, S.; Kornyshev, A. A. J. Chem. Phys. 2001, 114, 10039.

(26) Ando, K.; Hynes, J. T. J. Mol. Liq. 1995, 64, 25.

(27) Ando, K.; Hynes, J. T. J. Phys. Chem. B 1997, 101, 10464.

(28) Marx, D.; Tuckerman, M. E.; Hutter, J.; Parrinello, M. Nature 1999, 397, 601 .

(29) Zundel, G.; Fritsch, J. In The Chemical Physics of Solvation, Part B: Spectroscopy of Solvation; Dogonadze, R. R., Kálmán, E., Kornyshev, A. A., Ulstrup, J., Eds.; Elsevier: Amsterdam, 1986; p 21.

(30) Vuilleumier, R.; Borgis, D. J. Chem. Phys. 1999, 111, 4251.

(31) Schmitt, U. W.; Voth, G. A. J. Chem. Phys. 1999, 111, 9361.

(32) Kreuer, K.-D. In Proton Conductors; Colomban, P., Ed.; Cambridge University Press: Cambridge, 1992; p 474.

(33) Marcus, R. A.; Sutin, N. Biochim. Biophys. Acta 1985, 811, 265.

(34) Kuznetsov, A. M. Charge Transfer in Physics, Chemistry and Biology; Gordon \& Breach: Amsterdam, 1995; Chapter 6.

(35) Kuznetsov, A. M.; Ulstrup, J. Electron Transfer in Chemistry and Biology. An Introduction to the Theory; Wiley: Chichester, U.K., 1999; Chapter 10.

(36) van Kampen, N. G. Stochastic Processes in Physics and Chemistry; North-Holland: Amsterdam, 1984.

(37) Scheiner, S. Acc. Chem. Res. 1985, 18, 174

(38) Newton, M. D. J. Chem. Phys. 1977, 67, 5535.

(39) Hubbard, J. B.; Wolynes, P. G. In The Chemical Physics of Solvation. Part C: Solvation Phenomena in Specific Physical, Chemical, and Biological Systems; Dogonadze, R. R., Kálmán, E., Kornyshev, A. A., Ulstrup, J., Eds.; Elsevier: Amsterdam, 1988; p 21.

(40) Luth, K.; Scheiner, S. J. Chem. Phys. 1992, 97, 7507.

(41) Karlström, G. J. Phys. Chem. 1995, 92, 1315, 1318.

(42) Laasonen, K.; Klein, M. L. J. Phys. Chem. 1994, 98, 10079.

(43) Wei, D.; Salahub, D. R. J. Chem. Phys. 1997, 106, 6086

(44) Ojamae, L.; Shavitt, I.; Singer, S. J. J. Chem. Phys. 1998, 109, 5547.

(45) Landau, L. D.; Lifshitz, E. M. Quantum Mechanics, Pergamon: Oxford, 1965

(46) Proton Transfer in Hydrogen-Bonded Systems; Bountis, T., Ed.; NATO ASI Series B: Physics, 291; Plenum Press: New York, 1992.

(47) Vuilleumier, R.; Borgis, D. J. Phys. Chem. B 1998, 102, 4261

(48) Vuilleumier, R.; Borgis, D. Chem. Phys. Lett. 1998, 284, 71.

(49) Schmitt, U. W.; Voth, G. A. J. Phys. Chem. B 1998, 102, 5547

(50) Bell, R. P. The Proton in Chemistry, 2nd ed.; Chapman and Hall: London, 1973.

(51) Proton-Transfer Reactions; Caldin, E. F., Gold, V., Eds.; Chapman and Hall: London, 1975.

(52) Bell, R. P. The Tunnel Effect in Chemistry; Chapman and Hall: London, 1980.

(53) Tada, Y.; Ueno, M.; Tsuchihashi, N.; Shimizu, K. J. Sol. Chem. 1992, 59, 971

(54) Baumann, E. W. J. Chem. Eng. Data 1993, 38, 12

(55) Agmon, N. J. Chim. Phys.-Chim. Biol. 1996, 93, 1714.

(56) Levich, V. G.; Dogonadze, R. R.; German, E. D.; Kuznetsov, A. M.; Kharkats, Yu. I. Electrochem. Acta 1970, 15, 353.

(57) Saveant, J.-M. J. Am. Chem. Soc. 1987, 109, 6788.

(58) Marcus, R. A. J. Phys. Chem. A 1997, 101, 4072.

(59) German, E. D.; Kuznetsov, A. M. J. Phys. Chem. 1994, 98, 6120.

(60) Kuznetsov, A. M. J. Phys. Chem. A 1999, 103, 1239. 